\title{
A numerical homogenization method for heterogeneous, anisotropic elastic media based on multiscale theory
}

\author{
Kai Gao ${ }^{1}$, Eric T. Chung ${ }^{2}$, Richard L. Gibson, Jr. ${ }^{3}$, Shubin Fu ${ }^{4}$, and Yalchin Efendiev ${ }^{5}$
}

\begin{abstract}
The development of reliable methods for upscaling finescale models of elastic media has long been an important topic for rock physics and applied seismology. Several effective medium theories have been developed to provide elastic parameters for materials such as finely layered media or randomly oriented or aligned fractures. In such cases, the analytic solutions for upscaled properties can be used for accurate prediction of wave propagation. However, such theories cannot be applied directly to homogenize elastic media with more complex, arbitrary spatial heterogeneity. Therefore, we have proposed a numerical homogenization algorithm based on multiscale finite-element methods for simulating elastic wave propagation in heterogeneous, anisotropic elastic media. Specifically, our method used multiscale basis functions obtained
\end{abstract}

from a local linear elasticity problem with appropriately defined boundary conditions. Homogenized, effective medium parameters were then computed using these basis functions, and the approach applied a numerical discretization that was similar to the rotated staggered-grid finite-difference scheme. Comparisons of the results from our method and from conventional, analytical approaches for finely layered media showed that the homogenization reliably estimated elastic parameters for this simple geometry. Additional tests examined anisotropic models with arbitrary spatial heterogeneity in which the average size of the heterogeneities ranged from several centimeters to several meters, and the ratio between the dominant wavelength and the average size of the arbitrary heterogeneities ranged from 10 to 100 . Comparisons to finite-difference simulations proved that the numerical homogenization was equally accurate for these complex cases.

\section{INTRODUCTION}

Earth models for applications in seismic modeling or imaging in oil and gas exploration are often simplified to represent complex heterogeneity with smoothly changing physical properties, or sometimes layered media. A specific example in reservoir characterization is the representation of fractured media as a combination of an unfractured rock matrix and randomly or preferentially oriented fractures. Such approximations and simplifications provide a useful approach to include the influence of microscale heterogeneity in analysis of macroscale earth media. One of the fundamental problems is then how to define a set of equivalent medium parameters that can accurately reproduce the macroscale behavior of real rocks, including such properties as seismic velocity, density, and anisotropy, for instance.

Many methods have been applied to this problem, most of which are based on a horizontal layering approximation of the earth. Backus (1962) proposes an averaging method (Backus averaging) that averages the stress and displacement components in the vertical direction to estimate the properties of an equivalent elastic medium. This method has become the most widely applied method in practice. However, it considers only anisotropy up to transverse isotropy (TI) with a vertical axis (VTI) or a horizontal axis (HTI), whereas real geologic materials may exhibit more complicated anisotropy, such as TI with a titled axis (TTI), or monoclinic anisotropy (e.g., Tsvankin et al., 2010). The Backus-averaging method is extended to include lower symmet-

Manuscript received by the Editor 7 August 2014; revised manuscript received 17 March 2015; published online 5 June 2015.

${ }^{1}$ Formerly Texas A\&M University, Department of Geology and Geophysics, College Station, Texas, USA; presently Geophysics Group, Los Alamos National Laboratory, Los Alamos, New Mexico, USA. E-mail: kaigao87@gmail.com.

${ }^{2}$ The Chinese University of Hong Kong (CUHK), Department of Mathematics, Shatin, Hong Kong. E-mail: tschung@math.cuhk.edu.hk.

${ }^{3}$ Texas A\&M University, Department of Geology and Geophysics, College Station, Texas, USA. E-mail: gibson@tamu.edu.

${ }^{4}$ Texas A\&M University, Department of Mathematics, College Station, Texas, USA. E-mail: shubinfu89@gmail.com.

${ }^{5}$ Texas A\&M University, Department of Mathematics, College Station, Texas, USA and King Abdullah University of Science and Technology, Numerical Porous Media SRI Center (NumPor), Thuwal, Saudi Arabia. E-mail: efendiev@math.tamu.edu.

(C) 2015 Society of Exploration Geophysicists. All rights reserved. 
rical anisotropy by Helbig and Schoenberg (1987). Later, by applying matrix and group theory, Schoenberg and Muir (1989) develop a more general effective medium theory for horizontally aligned elastic layers, with general anisotropy in which all 21 independent elasticity constants might be nonzero in the elasticity matrix (we will refer to this method as the Schoenberg-Muir theory). Carcione et al. (2012) verify the Schoenberg-Muir theory by comparing the analytical solutions and the solutions calculated with a finite-difference method for the elastic wave equation, and they find that the Schoenberg-Muir theory can accurately determine effective elasticity parameters for finely layered elastic VTI, HTI, and TTI media as well.

A limitation of Backus averaging and the Schoenberg-Muir theory is that the fine-scale medium must be horizontally layered. Researchers have made some attempts to extend upscaling for media with general heterogeneities on the fine scale. For example, Rijpsma and Zijl (1998) and Zijl et al. (2002) propose a numerical homogenization procedure for Hooke's law, and they propose that the upscaling can be implemented by displacement-stress averaging, displacement-energy averaging, or stress-energy averaging. Grechka (2003) demonstrates another numerical method with more straightforward boundary conditions to solve the local problem. In both of these two methods, they solve an appropriately defined local problem, which is either the static or frequency-dependent equation of motion in the elastic medium. Given the solution of the local problem, the displacement, strain, or stress field can be averaged, and the effective elasticity tensor can be defined by assuming that the elastic wave equation has the same formulation on the coarse scale as that on the fine scale. These approaches are similar to Backus averaging, except that they are numerical; i.e., a local problem has to be solved numerically before the averaging.

Anisotropy of elastic properties can also come from preferentially aligned fractures, and there have been corresponding effective medium theories to quantitatively describe fractured rocks (e.g., Sayers and Kachanov, 1991; Schoenberg and Sayers, 1995; Sayers, 1996, 2002; Grechka and Kachanov, 2006; Tsvankin and Grechka, 2011). The overall properties of a cracked solid have also been studied by assuming circular or elliptic cracks (e.g., Budiansky and O'Connell, 1976; Hudson, 1980; Kachanov, 1980, 1992). These analysis approaches assume idealized geometries and orientations of fractures, but in this paper, we concentrate on media that do not require these assumptions and allow general distributions of elastic constants and density. Therefore, there are fundamental differences between these types of effective medium theories and the numerical approach we investigate in this paper.

From the aspect of numerical simulation of seismic wave propagation, the effective medium theory can be viewed as an approach to reduce the computational costs for wave equation modeling because the computational costs of various numerical methods for fullwavefield modeling, such as the finite-difference method (e.g., Dablain, 1986; Virieux, 1986) and the finite-element method (FEM) (e.g., Marfurt, 1984; Komatitsch et al., 1999; Käser and Dumbser, 2008), are directly proportional to the number of elements in the geologic models, and effective medium theory can provide a set of equivalent parameters that enables the simulations to be implemented with coarser elements. This problem has also been addressed by the so-called multiscale method for wave equations (Vdovina et al., 2005; Korostyshevskaya and Minkoff, 2006; Engquist et al., 2007; Owhadi and Zhang, 2008; Vdovina and Minkoff, 2008, 2011; Abdulle and Grote, 2011; Chung et al., 2011b, 2013;
Fu et al., 2013; Gao et al., 2013; Gibson et al., 2014). These various approaches to the multiscale problem can be quite different in their underlying principles, but they tend to reach one specific goal, that is, to solve the wave equations on a set of coarsely discretized mesh to approximate the solutions of the wave equations on the finely discretized mesh, and each coarse element may contain finer elements with highly heterogeneous medium properties in space. Compared with the effective medium theories that are derived with assumptions of idealized geometries (e.g., Backus, 1962), there are no restrictions on subgrid medium parameter variations in the multiscale method, which means that the subgrid media can be arbitrarily heterogeneous.

In this paper, we investigate a numerical homogenization approach to derive the effective medium parameters for arbitrarily heterogeneous elastic media with general anisotropy based on the multiscale method for wave equations (Chung et al., 2011a, 2011b; Gao et al., 2013; Gibson et al., 2014). We first define a local problem to determine the multiscale basis functions, with boundary conditions that favor the application of rotated staggered-grid (RSG) finite-difference-like scheme (Saenger et al., 2000; Saenger and Bohlen, 2004), and finally, we calculate the effective elasticity parameters by using these multiscale basis functions. This numerical approach, a multiscale method, allows for arbitrary subgrid medium parameter variations. We remark that the local problem we solve to determine basis functions is essentially the same as that applied by Zijl et al. (2002) and Grechka (2003). However, they apply different boundary conditions, and their numerical procedures are designed to compute parameters based on average stresses and strains in a coarse block. In contrast, our method is based on a rotated, staggered-grid finite-difference approach, and the boundary conditions are designed to be consistent with this algorithm. Furthermore, instead of computing average stress and strain, we compute multiscale basis functions, and the numerical homogenized effective parameters are based on appropriate summations of these bases. Also, we solve the local problem only once, whereas in Grechka (2003), the local problems have to be solved several times with different boundary conditions, which makes our approach a little more efficient. For these reasons, we call our derived effective medium parameters the numerical homogenized effective medium parameters, or simply, the homogenized parameters, to differentiate them from the effective medium parameters calculated with either an analytical approach or the stress-strain averaging approach.

Our paper will be organized as follows: We begin with the elastic wave equation in stress-velocity form and use this form to define the appropriate local problem and boundary conditions to obtain the multiscale basis functions. We next show how to calculate the homogenized medium parameters given these basis functions. In the third part, we present several numerical experiments to verify the effectiveness of our method. In addition, the appendices outline how to calculate the multiscale basis functions with second-order FEM, as well as 3D extensions of our method.

\section{THEORY}

\section{Elastic wave equation}

We start from the 2D elastic wave equation expressed in the stress-velocity form (Carcione, 2007):

$$
\partial_{t} \boldsymbol{\sigma}=\mathbf{C} \boldsymbol{\Lambda}^{\mathrm{T}} \mathbf{v}
$$


and

$$
\rho \partial_{t} \mathbf{v}=\Lambda \boldsymbol{\sigma}+\mathbf{f}
$$

where $\mathbf{v}=\mathbf{v}(\mathbf{x}, t)=\left(v_{1}, v_{3}\right)^{\mathrm{T}}$ is the particle velocity vector, $\boldsymbol{\sigma}=\boldsymbol{\sigma}(\mathbf{x}, t)=\left(\sigma_{11}, \sigma_{33}, \sigma_{13}\right)^{\mathrm{T}}$ is the stress tensor, $\mathbf{f}=\mathbf{f}(\mathbf{x}, t)=$ $\left(f_{1}, f_{3}\right)^{\mathrm{T}}$ is the external source term, and

$$
\mathbf{C}=\left(\begin{array}{lll}
C_{11} & C_{13} & C_{15} \\
C_{13} & C_{33} & C_{35} \\
C_{15} & C_{35} & C_{55}
\end{array}\right)
$$

is the elasticity matrix in Voigt notation, and we write the differential operator $\boldsymbol{\Lambda}$ as

$$
\boldsymbol{\Lambda}=\left(\begin{array}{ccc}
\partial_{1} & 0 & \partial_{3} \\
0 & \partial_{3} & \partial_{1}
\end{array}\right)
$$

Equation 1 can describe wave propagation in anisotropic elastic media with symmetry up to hexagonal anisotropy with tilted symmetry axis in the $x_{1}-x_{3}$-plane, i.e., TTI, and monoclinic anisotropy (assuming the symmetry plane is the $x_{1}-x_{3}$-plane), in which $C_{15}$ and $C_{35}$ are possibly nonzero. In the following analysis, we will omit the source term $\mathbf{f}$ for convenience.

\section{Multiscale basis function}

We discretize the computational domain $\Omega$ with a set of coarse mesh cells $\mathcal{Q}_{H}$ indicated by the black lines and black dashed lines in Figure 1. The support of $\boldsymbol{\sigma}$ is denoted by $K_{\sigma}$, and the support of $\mathbf{v}$ is denoted by $K_{v}$. Each coarse element $K_{\sigma}$ or $K_{v}$ in $\mathcal{Q}_{H}$ may contain finer elements, consisting of a finer discretization of $\Omega$, say $\mathcal{Q}_{h}$ indicated by the gray lines in Figure 1.

The goal of our multiscale approach to solve the numerical homogenization problem for arbitrary heterogeneous media is to derive a finite-difference-like scheme that can solve wave equation 1 on the coarse mesh $\mathcal{Q}_{H}$. This allows us to get a set of coefficients for the finite-difference terms that are equivalent to effective elastic parameters of the coarse elements.

We first express the stress wavefield on $\mathcal{Q}_{H}$ as

$$
\boldsymbol{\sigma}(\mathbf{x}, t)=\sum_{i}\left(p_{11, i} \phi_{11, i}, p_{33, i} \phi_{33, i}, p_{13, i} \phi_{13, i}\right),
$$

where $i$ represents the $i$ th coarse cell, $\phi_{k l, i}$ is the spatial multiscale basis function, and $p_{k l, i}$ is the temporal part of $\sigma_{k l}(\mathbf{x}, t)$ in the coarse cell. Rather than the conventional polynomial basis functions defined for FEM (e.g., Hughes, 1987; Larson and Bengzon, 2013), the multiscale basis functions here are determined through an appropriately defined local problem, which we will discuss later.

We also define the particle velocity wavefield on $\mathcal{Q}_{H}$ as

$$
\mathbf{v}(\mathbf{x}, t)=\sum_{i}\left(q_{1, i} \psi_{1, i}, q_{3, i} \psi_{3, i}\right),
$$

where we assume that the basis function $\psi_{k, i}$ is constant within each coarse cell, i.e., $\left(\psi_{1, i}, \psi_{3, i}\right)=(1,1)$, and $q_{k, i}$ is the temporal part of $v_{k}(\mathbf{x}, t)$ in the coarse cell. We also assume that the particle velocity components are weakly differentiable, so we can take $\partial_{l} v_{k}$ as the first-order weak derivative of $v_{k}$ along the $x_{l}$-direction. This assumption implies that $v_{k}$ can be integrated even though the derivative of the piecewise constant function is not well defined.
This mesh $\mathcal{Q}_{H}$ is similar to grid point positions in the RSG finitedifference scheme (Saenger et al., 2000; Saenger and Bohlen, 2004), in which the stress components are placed at the center of a grid, occupying integer-grid positions along both axes, and the velocity components are placed at the corners of a grid, occupying half-grid positions along both axes.

Now we define a local problem to calculate the multiscale basis functions $\phi_{i}(\mathbf{x})$, which is a linear elasticity extension of the multiscale basis functions in the so-called multiscale FEM (MsFEM) method developed for the acoustic case (Efendiev and Hou, 2009; Chung et al., 2011a, 2011b; Gibson et al., 2014).

We know that $K_{\sigma}=\left[i_{1}-1 / 2, i_{1}+1 / 2\right] \times\left[i_{3}-1 / 2, i_{3}+1 / 2\right]$ for stress $\boldsymbol{\sigma}\left(i_{1}, i_{3}\right)$ is in fact also composed of four subrectangles, which are parts of the support of $\mathbf{v}\left(i_{1}-1 / 2, i_{3}-1 / 2\right)$, $\mathbf{v}\left(i_{1}+1 / 2, i_{3}-1 / 2\right), \mathbf{v}\left(i_{1}-1 / 2, i_{3}+1 / 2\right)$, and $\mathbf{v}\left(i_{1}+1 / 2, i_{3}+1 / 2\right)$, as indicated in Figure 1. When determining the multiscale basis functions for $\boldsymbol{\sigma}\left(i_{1}, i_{3}\right)$, we solve the following local static linear elasticity problem in each of these four subrectangles:

$$
\boldsymbol{\sigma}=\mathbf{C} \boldsymbol{\Lambda}^{\mathrm{T}} \mathbf{u}, \quad-\boldsymbol{\Lambda} \boldsymbol{\sigma}=\mathbf{0},
$$

under the boundary conditions

$$
\begin{gathered}
\sigma_{11}=1, \quad \text { on } \mathcal{E}_{1}, \quad \sigma_{33}=1, \quad \text { on } \mathcal{E}_{3}, \\
\sigma_{13}=1, \quad \text { on } \mathcal{E}_{1} \text { and } \mathcal{E}_{3},
\end{gathered}
$$

where $\mathcal{E}_{1}$ and $\mathcal{E}_{3}$ are the two vertical and horizontal edges of each of the four subrectangles, respectively, and $\mathbf{u}$ is the displacement. The local problem 6 along with the boundary conditions 7 can be solved with the second-order FEM for the linear elasticity problem (e.g., Larson and Bengzon, 2013). We present the necessary details for this solution in Appendix A. Solutions of $\sigma_{11}, \sigma_{33}$, and $\sigma_{13}$, which are denoted as $\phi_{11}, \phi_{33}$, and $\phi_{13}$, in each of these four rectangles, are joined in $K_{\sigma}$ and taken as the multiscale basis functions of $\sigma_{11}\left(i_{1}, i_{3}\right), \sigma_{33}\left(i_{1}, i_{3}\right)$ and $\sigma_{13}\left(i_{1}, i_{3}\right)$, respectively.

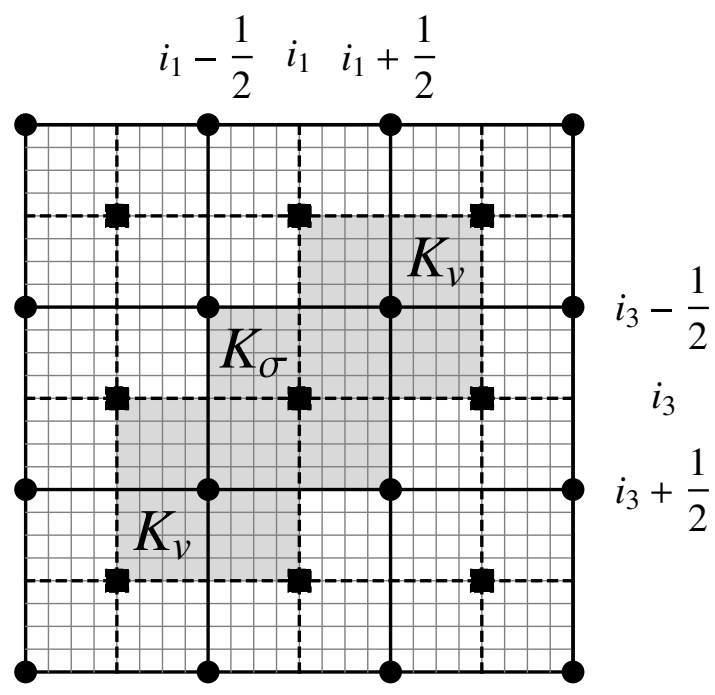

Figure 1. The mesh discretization of domain $\Omega$, where $\mathbf{m}$ represents $\boldsymbol{\sigma}=\left(\sigma_{11}, \sigma_{33}, \sigma_{13}\right), \bullet$ represents $\mathbf{v}=\left(v_{1}, v_{3}\right)$, the black line rectangle $K_{\sigma}$ represents the support of stress components, and the black dashed line rectangle $K_{v}$ represents the support of velocity components. 


\section{Homogenized medium parameters}

We then transform equation 1a into an equivalent form as

$$
\mathbf{S} \partial_{t} \boldsymbol{\sigma}=\boldsymbol{\Lambda}^{\mathrm{T}} \mathbf{v}
$$

where $\mathbf{S}=\mathbf{C}^{-1}$. Explicitly, it is,

$$
\left(\begin{array}{lll}
S_{11} & S_{13} & S_{15} \\
S_{13} & S_{33} & S_{35} \\
S_{15} & S_{35} & S_{55}
\end{array}\right) \partial_{t}\left(\begin{array}{c}
\sigma_{11} \\
\sigma_{33} \\
\sigma_{13}
\end{array}\right)=\left(\begin{array}{c}
\partial_{1} v_{1} \\
\partial_{3} v_{3} \\
\partial_{1} v_{3}+\partial_{3} v_{1}
\end{array}\right) .
$$

Beginning with the first equation 9, we write the stress as the product of the spatial basis function and temporal function as defined in equation 4, multiply both sides by a test function $\phi_{11}$, and integrate over the support $K_{\sigma}$ of stress components $\boldsymbol{\sigma}\left(i_{1}, i_{3}\right)$ to get

$$
\begin{aligned}
& \left(\int_{K_{\sigma}} S_{11} \phi_{11} \phi_{11} \mathrm{~d} \mathbf{x} \int_{K_{\sigma}} S_{13} \phi_{33} \phi_{11} \mathrm{~d} \mathbf{x} \int_{K_{\sigma}} S_{15} \phi_{13} \phi_{11} d \mathbf{x}\right) \\
& \quad \times\left(\begin{array}{c}
\dot{p}_{11} \\
\dot{p}_{33} \\
\dot{p}_{13}
\end{array}\right)=\int_{K_{\sigma}} \phi_{11} \partial_{1} v_{1} \mathrm{~d} \mathbf{x}
\end{aligned}
$$

where $\dot{p}_{i j}=d p_{i j} / d t$. To get the homogenized medium parameters for $K_{\sigma}$, we need to eliminate the integral on both sides of the above equation. For the right side of equation 10 , we can integrate by parts:

$$
\begin{aligned}
\int_{K_{\sigma}} \phi_{11} \partial_{1} v_{1} \mathrm{~d} \mathbf{x}= & \int_{i_{3}-1 / 2}^{i_{3}+1 / 2} \int_{i_{1}-1 / 2}^{i_{1}+1 / 2} \phi_{11} \partial_{1} v_{1} \mathrm{~d} x_{1} \mathrm{~d} x_{3} \\
= & \left.\left(\int_{i_{3}-1 / 2}^{i_{3}+1 / 2} v_{1} \phi_{11} \mathrm{~d} x_{3}\right)\right|_{i_{1}-1 / 2} ^{i_{1}+1 / 2} \\
& -\int_{i_{3}-1 / 2}^{i_{3}+1 / 2} \int_{i_{1}-1 / 2}^{i_{1}+1 / 2} v_{1} \partial_{1} \phi_{11} \mathrm{~d} x_{1} \mathrm{~d} x_{3} .
\end{aligned}
$$

The above derivation applies the assumption that the velocity component is weakly differentiable so that $\partial_{1} v_{1}$ can be integrated.

Next, we simplify the second part in the right side of equation 11 . Recall that the velocity component $v_{1}$ is constant in each of the four rectangles composing of $K_{\sigma}\left(i_{1}, i_{3}\right)$. Therefore,

$$
\begin{aligned}
& \int_{i_{3}-1 / 2}^{i_{3}+1 / 2} \int_{i_{1}-1 / 2}^{i_{1}+1 / 2} v_{1} \partial_{1} \phi_{11} \mathrm{~d} x_{1} \mathrm{~d} x_{3} \\
& =\int_{i_{3}-1 / 2}^{i_{3}} \int_{i_{1}-1 / 2}^{i_{1}} v_{1} \partial_{1} \phi_{11} \mathrm{~d} x_{1} \mathrm{~d} x_{3} \\
& +\int_{i_{3}-1 / 2}^{i_{3}} \int_{i_{1}}^{i_{1}+1 / 2} v_{1} \partial_{1} \phi_{11} \mathrm{~d} x_{1} \mathrm{~d} x_{3} \\
& +\int_{i_{3}}^{i_{3}+1 / 2} \int_{i_{1}-1 / 2}^{i_{1}} v_{1} \partial_{1} \phi_{11} \mathrm{~d} x_{1} \mathrm{~d} x_{3} \\
& +\int_{i_{3}}^{i_{3}+1 / 2} \int_{i_{1}}^{i_{1}+1 / 2} v_{1} \partial_{1} \phi_{11} \mathrm{~d} x_{1} \mathrm{~d} x_{3} .
\end{aligned}
$$

Then, for each of the four rectangles, e.g., $K_{\sigma, 1}=\left[i_{1}-1 / 2, i_{1}\right] \times$ $\left[i_{3}-1 / 2, i_{3}\right]$ because $v_{1}$ is constant and $\phi_{11}=1$ on two vertical edges of $K_{\sigma, 1}$, we have

$$
\begin{aligned}
& \int_{i_{3}-1 / 2}^{i_{3}} \int_{i_{1}-1 / 2}^{i_{1}} v_{1} \partial_{1} \phi_{11} \mathrm{~d} x_{1} \mathrm{~d} x_{3} \\
& =v_{1}\left(i_{1}-\frac{1}{2}, i_{3}-\frac{1}{2}\right) \int_{i_{3}-1 / 2}^{i_{3}} \int_{i_{1}-1 / 2}^{i_{1}} \partial_{1} \phi_{11} \mathrm{~d} x_{1} \mathrm{~d} x_{3} \\
& \quad=v_{1}\left(i_{1}-\frac{1}{2}, i_{3}-\frac{1}{2}\right) \int_{i_{3}-1 / 2}^{i_{3}} \int_{1}^{1} d \phi_{11} \mathrm{~d} x_{3} \\
& =v_{1}\left(i_{1}-\frac{1}{2}, i_{3}-\frac{1}{2}\right) \times 0 \\
& =0 .
\end{aligned}
$$

For the other three integrals in equation 12 , we have the same result, and therefore

$$
\int_{i_{3}-1 / 2}^{i_{3}+1 / 2} \int_{i_{1}-1 / 2}^{i_{1}+1 / 2} v_{1} \partial_{1} \phi_{11} \mathrm{~d} x_{1} \mathrm{~d} x_{3}=0
$$

For the first part in equation 11 , we have

$$
\begin{aligned}
& \left.\left(\int_{i_{3}-1 / 2}^{i_{3}+1 / 2} v_{1} \phi_{11} \mathrm{~d} x_{3}\right)\right|_{i_{1}-1 / 2} ^{i_{1}+1 / 2} \\
& =\left.\left(\int_{i_{3}-1 / 2}^{i_{3}} v_{1} \phi_{11} \mathrm{~d} x_{3}+\int_{i_{3}}^{i_{3}+1 / 2} v_{1} \phi_{11} \mathrm{~d} x_{3}\right)\right|_{i_{1}-1 / 2} ^{i_{1}+1 / 2} \\
& =\int_{i_{3}-1 / 2}^{i_{3}}\left[v_{1}\left(i_{1}+\frac{1}{2}, x_{3}\right)-v_{1}\left(i_{1}-\frac{1}{2}, x_{3}\right)\right] \phi_{11} \mathrm{~d} x_{3} \\
& +\int_{i_{3}}^{i_{3}+1 / 2}\left[v_{1}\left(i_{1}+\frac{1}{2}, x_{3}\right)-v_{1}\left(i_{1}-\frac{1}{2}, x_{3}\right)\right] \phi_{11} \mathrm{~d} x_{3} \\
& =\frac{\Delta x_{3}}{2}\left[v_{1}\left(i_{1}+\frac{1}{2}, i_{3}+\frac{1}{2}\right)+v_{1}\left(i_{1}+\frac{1}{2}, i_{3}-\frac{1}{2}\right)\right. \\
& \left.-v_{1}\left(i_{1}-\frac{1}{2}, i_{3}+\frac{1}{2}\right)-v_{1}\left(i_{1}-\frac{1}{2}, i_{3}-\frac{1}{2}\right)\right] \\
& =\frac{\Delta r}{2 \Delta x_{1}} \frac{1}{\Delta r}\left\{\left[v_{1}\left(i_{1}+\frac{1}{2}, i_{3}+\frac{1}{2}\right)-v_{1}\left(i_{1}-\frac{1}{2}, i_{3}-\frac{1}{2}\right)\right]\right. \\
& \left.+\left[v_{1}\left(i_{1}+\frac{1}{2}, i_{3}-\frac{1}{2}\right)-v_{1}\left(i_{1}-\frac{1}{2}, i_{3}+\frac{1}{2}\right)\right]\right\} \Delta x_{1} \Delta x_{3} \\
& \approx
\end{aligned}
$$

where $\Delta r=\sqrt{\Delta x_{1}^{2}+\Delta x_{3}^{2}} ; D_{1}$ and $D_{3}$ are the partial derivatives along the rotated axes, as defined in the RSG finite-difference method (Saenger et al., 2000, 2007; Saenger and Bohlen, 2004); and the last step in equation 15 uses the discrete finite-difference term to approximate the continuous partial derivatives, and therefore

$$
\int_{K_{\sigma}} \phi_{11} \partial_{1} v_{1} \mathrm{~d} \mathbf{x} \approx \Delta x_{1} \Delta x_{3} \partial_{1} v_{1}=S_{K_{\sigma}} \partial_{1} v_{1},
$$

where $S_{K_{\sigma}}=\Delta x_{1} \Delta x_{3}$ is the area of $K_{\sigma}$.

Meanwhile, for the left side of equation 10, because both $S_{i j}$ and $\phi_{s t}$ are discrete values on each fine grid within $K_{\sigma}$, we have, for example, for rectangular grids inside the coarse cell, 


$$
\begin{aligned}
& \int_{K_{\sigma}} S_{11} \phi_{11} \phi_{11} \mathrm{~d} \mathbf{x} \\
& \approx \\
& \quad \frac{S_{K_{\sigma}}}{n_{1} n_{3}} \sum_{j_{1}=1}^{n_{1}} \sum_{j_{3}=1}^{n_{3}} S_{11}\left(j_{1}, j_{3}\right) \phi_{11}\left(j_{1}, j_{3}\right) \phi_{11}\left(j_{1}, j_{3}\right) \\
& =S_{K_{\sigma}} \tilde{S}_{11},
\end{aligned}
$$

where $n_{1}$ and $n_{3}$ represent the grid number in the $x_{1}$ - and $x_{3}$-directions within $K_{\sigma}$, respectively. Essentially, $\tilde{S}_{11}$ is the average of the integral on the left side of equation 17.

With the above results, for the first equation, we finally have

$$
\left(\begin{array}{lll}
\tilde{S}_{11} & \tilde{S}_{33} & \tilde{S}_{13}
\end{array}\right)\left(\begin{array}{c}
\dot{p}_{11} \\
\dot{p}_{33} \\
\dot{p}_{13}
\end{array}\right)=\partial_{1} v_{1},
$$

where $\tilde{S}_{i j}$ represents the homogenized compliance in $K_{\sigma}$, and it can be calculated as shown in equation 17. Equation 18 is defined on coarse mesh $\mathcal{Q}_{H}$ with effective elasticity parameters $\tilde{S}_{i j}$.

We can repeat the same manipulation for the second equation in equation 9. Based on the boundary conditions, we prescribe for $\phi_{33}$, i.e., $\phi_{33}=1$ on two horizontal edges of each of the four subrectangles, we integrate both sides with a test function $\phi_{33}$ on both sides, and then for the right side, we have

$$
\begin{aligned}
\int_{K_{\sigma}} \phi_{33} \partial_{3} v_{3} \mathrm{~d} \mathbf{x} & =\int_{i_{3}-1 / 2}^{i_{3}+1 / 2} \int_{i_{1}-1 / 2}^{i_{1}+1 / 2} \phi_{33} \partial_{3} v_{3} \mathrm{~d} x_{1} \mathrm{~d} x_{3} \\
& =\left.\left(\int_{i_{1}-1 / 2}^{i_{1}+1 / 2} v_{3} \phi_{33} \mathrm{~d} x_{1}\right)\right|_{i_{3}-1 / 2} ^{i_{3}+1 / 2} \\
& -\int_{i_{3}-1 / 2}^{i_{3}+1 / 2} \int_{i_{1}-1 / 2}^{i_{1}+1 / 2} v_{3} \partial_{3} \phi_{33} \mathrm{~d} x_{1} \mathrm{~d} x_{3} \\
& \approx S_{K_{\sigma}} \partial_{3} v_{3} .
\end{aligned}
$$

Again, the terms on the light side can be calculated similar to those in equation 17.

Finally, for the third equation in equation 9, we integrate both sides with a test function $\phi_{13}$ in $K_{\sigma}$ and obtain the right side as

$$
\begin{aligned}
\int_{K_{\sigma}} & \phi_{13}\left(\partial_{1} v_{3}+\partial_{3} v_{1}\right) \mathrm{d} \mathbf{x} \\
= & \int_{i_{3}-1 / 2}^{i_{3}+1 / 2} \int_{i_{1}-1 / 2}^{i_{1}+1 / 2}\left(\phi_{13} \partial_{1} v_{3}+\phi_{13} \partial_{3} v_{1}\right) \mathrm{d} x_{1} \mathrm{~d} x_{3} \\
= & \left.\left(\int_{i_{3}-1 / 2}^{i_{3}+1 / 2} v_{3} \phi_{13} \mathrm{~d} x_{3}\right)\right|_{i_{1}-1 / 2} ^{i_{1}+1 / 2} \\
- & \int_{i_{3}-1 / 2}^{i_{3}+1 / 2} \int_{i_{1}-1 / 2}^{i_{1}+1 / 2} v_{3} \partial_{1} \phi_{13} \mathrm{~d} x_{1} \mathrm{~d} x_{3} \\
+ & \left.\left(\int_{i_{1}-1 / 2}^{i_{1}+1 / 2} v_{1} \phi_{13} d x_{1}\right)\right|_{i_{3}-1 / 2} ^{i_{3}+1 / 2} \\
- & \int_{i_{3}-1 / 2}^{i_{3}+1 / 2} \int_{i_{1}-1 / 2}^{i_{1}+1 / 2} v_{1} \partial_{3} \phi_{13} \mathrm{~d} x_{1} \mathrm{~d} x_{3} \\
\approx & S_{K_{\sigma}}\left(\partial_{1} v_{3}+\partial_{3} v_{1}\right)
\end{aligned}
$$

and the left side can be obtained in a similar approach to that used for equation 17.

Now, we examine equation $1 \mathrm{~b}$. We integrate the first equation within $1 \mathrm{~b}$ in the support of $\mathbf{v}\left(i_{1}+1 / 2, i_{3}+1 / 2\right)$ and get

$$
\begin{aligned}
\int_{i_{3}}{ }^{i_{3}}+1 & \int_{i_{1}}^{i_{1}+1} \rho \partial_{t} v_{1} \mathrm{~d} x_{1} \mathrm{~d} x_{3} \\
= & \int_{i_{3}}^{i_{3}+1} \int_{i_{1}}^{i_{1}+1} \partial_{1} \sigma_{11} \mathrm{~d} x_{1} \mathrm{~d} x_{3}+\int_{i_{3}}^{i_{3}+1} \int_{i_{1}}^{i_{1}+1} \partial_{3} \sigma_{13} \mathrm{~d} x_{1} \mathrm{~d} x_{3} \\
= & \int_{i_{3}}^{i_{3}+1} \int_{\sigma_{11}\left(i_{1}, x_{3}\right)}^{\sigma_{11}\left(i_{1}+1, x_{3}\right)} \mathrm{d} \sigma_{11} \mathrm{~d} x_{3}+\int_{i_{1}}^{i_{1}+1} \int_{\sigma_{13}\left(x_{1}, i_{3}\right)}^{\sigma_{13}\left(x_{1}, i_{3}+1\right)} \mathrm{d} \sigma_{13} \mathrm{~d} x_{1} \\
= & \int_{i_{3}}^{i_{3}+1}\left[\sigma_{11}\left(i_{1}+1, x_{3}\right)-\sigma_{11}\left(i_{1}, x_{3}\right)\right] \mathrm{d} x_{3} \\
+ & \int_{i_{1}}^{i_{1}+1}\left[\sigma_{13}\left(x_{1}, i_{3}+1\right)-\sigma_{13}\left(x_{1}, i_{3}\right)\right] \mathrm{d} x_{1} \\
= & {\left[\int_{i_{3}}^{i_{3}+1 / 2} \sigma_{11}\left(i_{1}+1, x_{3}\right) \mathrm{d} x_{3}+\int_{i_{3}+1 / 2}^{i_{3}+1} \sigma_{11}\left(i_{1}+1, x_{3}\right) \mathrm{d} x_{3}\right] } \\
- & {\left[\int_{i_{3}}^{i_{3}+1 / 2} \sigma_{11}\left(i_{1}, x_{3}\right) \mathrm{d} x_{3}+\int_{i_{3}+1 / 2}^{i_{3}+1} \sigma_{11}\left(i_{1}, x_{3}\right) \mathrm{d} x_{3}\right] } \\
+ & {\left[\int_{i_{1}}^{i_{1}+1 / 2} \sigma_{13}\left(x_{1}, i_{3}+1\right) \mathrm{d} x_{1}+\int_{i_{1}+1 / 2}^{i_{1}+1} \sigma_{13}\left(x_{1}, i_{3}+1\right) \mathrm{d} x_{1}\right] } \\
+ & {\left[\int_{i_{1}}^{i_{1}+1 / 2} \sigma_{13}\left(x_{1}, i_{3}\right) \mathrm{d} x_{1}+\int_{i_{1}+1 / 2}^{i_{1}+1} \sigma_{13}\left(x_{1}, i_{3}\right) d x_{1}\right] } \\
= & \frac{\Delta x_{3}}{2}\left[\sigma_{11}\left(i_{1}+1, i_{3}\right)+\sigma_{11}\left(i_{1}+1, i_{3}+1\right)\right. \\
- & \left.\sigma_{13}\left(i_{1}, i_{3}\right)-\sigma_{11}\left(i_{1}, i_{3}+1\right)\right] \\
& \Delta \sigma_{13}\left(i_{1}, i_{3}+1\right)+\sigma_{13}\left(i_{1}+1, i_{3}+1\right) \\
&
\end{aligned}
$$

Recalling the manipulations in the development of equation 15 , it is straightforward to find that

$$
\begin{aligned}
& \int_{i_{3}}^{i_{3}+1} \int_{i_{1}}^{i_{1}+1} \rho \partial_{t} v_{1} \mathrm{~d} x_{1} \mathrm{~d} x_{3} \\
& \approx \\
& \approx \frac{\Delta r}{2 \Delta x_{1}}\left(D_{3} \sigma_{11}+D_{1} \sigma_{11}\right) \Delta x_{1} \Delta x_{3} \\
& \quad+\frac{\Delta r}{2 \Delta x_{3}}\left(D_{3} \sigma_{13}-D_{1} \sigma_{13}\right) \Delta x_{1} \Delta x_{3} \\
& \quad=S_{K_{v}}\left(\partial_{1} \sigma_{11}+\partial_{3} \sigma_{13}\right)
\end{aligned}
$$

where $S_{K}$ is the area of the support of $\mathbf{v}\left(i_{1}+1 / 2, i_{3}+1 / 2\right)$. Furthermore, for the left side of equation 22 because $v_{1}$ is constant in $K_{v}$, we then have

$$
\begin{aligned}
\int_{i_{3}}^{i_{3}+1} \int_{i_{1}}^{i_{1}+1} \rho \partial_{t} v_{1} \mathrm{~d} x_{1} \mathrm{~d} x_{3} & =\partial_{t} v_{1} \int_{i_{3}}^{i_{3}+1} \int_{i_{1}}^{i_{1}+1} \rho \mathrm{d} x_{1} \mathrm{~d} x_{3} \\
& \approx \partial_{t} v_{1} \tilde{\rho} S_{K_{v}},
\end{aligned}
$$


with

$$
\tilde{\rho}=\frac{1}{m_{1} m_{3}} \sum_{j_{1}=1}^{m_{1}} \sum_{j_{3}=1}^{m_{3}} \rho\left(j_{1}, j_{3}\right)
$$

Again, $\tilde{\rho}$ is the average of the integral on the right side of equation 24 , which is similar to the homogenized compliance in equation 17. It is not surprising that the homogenized mass density is the area average of individual density values in $2 \mathrm{D}$, but this result shows that the method is determining correct effective medium properties; therefore,

$$
\tilde{\rho} \partial_{t} v_{1}=\partial_{1} \sigma_{11}+\partial_{3} \sigma_{13}
$$

Similarly, for the second equation $1 \mathrm{~b}$, we have

$$
\tilde{\rho} \partial_{t} v_{3}=\partial_{1} \sigma_{13}+\partial_{3} \sigma_{33}
$$

with $\tilde{\rho}$ having exactly the same definition as that in equation 24 .

We then arrive at the homogenized elastic wave equation on the coarse mesh $\mathcal{Q}_{H}$ as follows:

$$
\partial_{t} \boldsymbol{\sigma}=\tilde{\mathbf{C}} \boldsymbol{\Lambda}^{\mathrm{T}} \mathbf{v}
$$

and

$$
\tilde{\rho} \partial_{t} \mathbf{v}=\Lambda \boldsymbol{\sigma}+\mathbf{f}
$$

where the homogenized elasticity matrix $\tilde{\mathbf{C}}=\tilde{\mathbf{S}}^{-1}$, and

$$
\tilde{\mathbf{S}}=\left(\begin{array}{ccc}
S_{11} \phi_{11} \phi_{11} & S_{13} \phi_{33} \phi_{11} & S_{15} \phi_{13} \phi_{11} \\
S_{13} \phi_{11} \phi_{33} & S_{33} \phi_{33} \phi_{33} & S_{35} \phi_{13} \phi_{33} \\
S_{15} \phi_{11} \phi_{13} & S_{35} \phi_{33} \phi_{13} & S_{55} \phi_{13} \phi_{13}
\end{array}\right)
$$

where each of the elements in $\tilde{\mathbf{S}}$ is a summation of the product of compliances and basis functions of all the fine elements within $K_{\sigma}$ given by

$$
S_{i j} \phi_{s t} \phi_{p q}=\frac{1}{n_{1} n_{3}} \sum_{j_{1}=1}^{n_{1}} \sum_{j_{3}=1}^{n_{3}} S_{i j}\left(j_{1}, j_{3}\right) \phi_{s t}\left(j_{1}, j_{3}\right) \phi_{p q}\left(j_{1}, j_{3}\right) .
$$

The homogenized density is computed with equation 24 . The above results are valid for rectangular fine grids within the coarse cell. For fine elements with general shapes, the homogenized parameters are the average of the corresponding integrals expressed in equations 17 and 23.

Because each $\tilde{\mathbf{C}}$ is computed as a weighted average of compliances, it can be interpreted as an effective property that is in some ways analogous to Backus averaging. In our case, however, the weight terms in the averaging are the basis function terms computed numerically from the solution of the local elasticity problem.

This approach does not impose any restrictions on the geometry or magnitude of subgrid medium variations, which therefore can be arbitrary. In such cases, even for simple layered subgrid medium property variations, there are no analytic results for $\phi_{11}, \phi_{33}$, or $\phi_{13}$; i.e., they can be only determined numerically, and this is quite different from previous theories such as Backus averaging or the Schoenberg-Muir theory. Also, this method can be straightforwardly extended to the 3D case (see Appendix B) in which general anisotropy with all 21 independent elasticity constants is addressed, and, again, the subgrid medium can be arbitrarily heterogeneous.

\section{NUMERICAL RESULTS}

We perform three kinds of numerical tests to verify the accuracy of our proposed method. The test results are compared with those obtained using Backus averaging and the Schoenberg-Muir theory, as well as the results calculated with a finite-difference method for elastic wave equations in which Backus averaging or the Schoenberg-Muir theory cannot be applied. For clarity, when we show the elasticity matrices in the following text, we only write the upper triangle part, noting here that they are all symmetric matrices.

\section{Horizontally layered medium}

In the first set of tests, we compare the results from Backus averaging, the Schoenberg-Muir theory, and our multiscale method for horizontally layered isotopic and anisotropic elastic media. It is important to note that the original version of Backus averaging method is valid for describing the effective properties of media composed of isotropic elastic layers, or elastic layers with anisotropy up to VTI and HTI; i.e., $C_{15}=C_{35}=0$. Schoenberg-Muir theory can describe the effective properties of media composed of layers with generally anisotropic elastic properties, including TTI or triclinic anisotropy in which all 21 independent elasticity constants are nonzero. Here, we restrict our attention to anisotropy up to 2D TTI, so that medium properties can be described by the elasticity matrix in equation 2 .

For simplicity, we take the two sets of test parameters presented by Carcione et al. (2012). In their tests, they use the SchoenbergMuir theory to derive the effective medium parameters and verify the accuracy by comparing the spatial wavefields calculated by the Fourier pseudospectral method for the original layered medium and the effective medium.

The first test is for a medium composed of VTI layers and HTI layers, with elastic constants $C_{11}=46.00, C_{13}=18.00$, $C_{15}=0.00, C_{33}=30.00, C_{35}=0.00$, and $C_{55}=7.00$ for the VTI layers and $C_{11}=30.00, C_{13}=18.00, C_{15}=0.00, C_{33}=$ $46.00, C_{35}=0.00$, and $C_{55}=7.00$ for HTI layers. We assume that the fine-grid model contains $1000 \times 1000$ grids, and we set the coarsening to be $10 \times 10$, which means that in each coarse-grid block, there are $10 \times 10$ fine grids. The size of the fine grid is $1 \mathrm{~m}$ in each direction, and in our test, we set the layer thickness to $1 \mathrm{~m}$ as well.

The effective elasticity constants from Backus averaging or Schoenberg-Muir theory are given as (Carcione et al., 2012)

$$
\mathbf{C}_{\text {Backus }}=\mathbf{C}_{\text {Schoenberg-Muir }}=\left(\begin{array}{ccc}
38.00 & 18.00 & 0 \\
& 36.30 & 0 \\
& & 7.00
\end{array}\right) \mathrm{GPa},
$$

and by our method, they are given as 


$$
\mathbf{C}_{\text {multiscale }}=\left(\begin{array}{ccc}
37.78 & 17.89 & 0 \\
& 36.24 & 0 \\
& & 7.00
\end{array}\right) \mathrm{GPa} .
$$

Because Carcione et al. (2012) verify the results of the Schoenberg-Muir theory by means of wave equation modeling, we take the results from Schoenberg-Muir theory to be the "true" solution. We compare the results from Backus averaging, Schoenberg-Muir theory, and our method, and we see that for such a layer composition, Backus averaging and Schoenberg-Muir theory give exactly the same result, and due to the numerical solution nature of our method, we obtain slightly different values. In fact, the relative differences of the elasticity constants from our calculated parameters, defined as $\mathbf{e}=e_{i j}=\left(C_{i j \text {,multiscale }}-C_{i j}\right) / C_{i j}$, with $C_{i j}$ being the effective elasticity constants solved from Backus averaging or the Schoenberg-Muir theory, are shown as

$$
\mathbf{e}=-\left(\begin{array}{lll}
0.57 \% & 0.62 \% & 0 \\
& 0.15 \% & 0 \\
& & 0
\end{array}\right)
$$

In the second test, the medium is composed of horizontal VTI and TTI layers. The layer thickness is again $1 \mathrm{~m}$. The elasticity constants are $C_{11}=46.00, C_{13}=18.00, C_{15}=0.00, C_{33}=30.00$, $C_{35}=0.00$, and $C_{55}=7.00$ for the VTI medium and $C_{11}=$ $35.00, \quad C_{13}=21.00, C_{15}=-4.00, \quad C_{33}=35.00, \quad C_{35}=-4.00$, and $C_{55}=10.00$ for the TTI material, which has a symmetry axis oriented at $45^{\circ}$ with respect to the layering. In this case, we can only compare the Schoenberg-Muir theory and our method to test results. The Schoenberg-Muir solution is

$$
\mathbf{C}_{\text {Schoenberg-Muir }}=\left(\begin{array}{ccc}
40.00 & 19 & -1.6 \\
& 31.90 & -1.5 \\
& & 8.1
\end{array}\right) \mathrm{GPa} \text {, }
$$

and our solution is

$$
\mathbf{C}_{\text {multiscale }}=\left(\begin{array}{ccc}
39.83 & 18.85 & -1.58 \\
& 31.50 & -1.43 \\
& & 8.11
\end{array}\right) \mathrm{GPa},
$$

with relative differences

$$
\mathbf{e}=-\left(\begin{array}{ccc}
0.42 \% & 0.80 \% & 1.44 \% \\
& 1.26 \% & 4.64 \% \\
& & -0.20 \%
\end{array}\right)
$$

Except for $C_{15}, C_{33}$, and $C_{35}$, the parameters have less than $1 \%$ relative error. The difference for $C_{35}$ between our approach and analytical result is relatively large compared with that of the other elasticity constants, and it is not obvious why this constant is unique. This might be due to numerical errors introduced when we solve the local problem because we use only second-order FEM. By improving the accuracy of the scheme of solving the local problem, this difference might be reduced.

\section{Arbitrarily heterogeneous media}

In the proceeding section, we compare our method with Backus averaging and Schoenberg-Muir theory and see that our method can be accurate. However, as we have discussed before, neither the Backus method nor Schoenberg-Muir theory is directly applicable to media that are arbitrarily heterogeneous, whereas there are no such difficulties for our method. In the following, we will apply our method to such a model, and because there is no independent solution in this situation, we will compare the wave equation modeling results from the fine-grid model and from the homogenized medium. For both cases, we use the 20th-order RSG finite-difference method (see Appendix C) to simulate the elastic wavefield propagation.

The first model is composed of $200 \times 200$ coarse elements, each of which is composed of $20 \times 20$ fine elements, and each of the fine elements has a size of $1 \mathrm{~m} \times 1 \mathrm{~m}$. Figure $2 \mathrm{a}-2 \mathrm{f}$ shows the elasticity parameter variations within each coarse element. All elasticity constants show some heterogeneities, including horizontal layering, elliptic inclusions with different orientations, and random heterogeneities as well. Clearly, such a subgrid model cannot be treated as a finely layered model, and therefore, Backus averaging or Schoenberg-Muir theory cannot be directly used here. The elasticity parameters for the numerical homogenized medium can be computed using equation 29 giving

$$
\mathbf{C}_{\text {multiscale }}=\left(\begin{array}{ccc}
38.96 & 20.40 & -1.21 \\
& 34.52 & -0.96 \\
& & 8.92
\end{array}\right) \mathrm{GPa} \text {, }
$$

which represents monoclinic anisotropy. We assume constant density for the model; i.e., $\rho=2500 \mathrm{~kg} / \mathrm{m}^{3}$. Thus, the $\mathrm{qP}$ - and $\mathrm{qSV}$ wave velocities are about $3.95 \times 10^{3}$ and $1.88 \times 10^{3} \mathrm{~m} / \mathrm{s}$ along the $x_{1}$-axis, respectively, and $3.72 \times 10^{3}$ and $1.89 \times 10^{3} \mathrm{~m} / \mathrm{s}$ along the $x_{3}$-axis. This test is for a $2 \mathrm{D}$ case in which the subgrid variations of elasticity parameters can be represented by $C_{I J}$ with $I, J=1,3,5$. If the model is $3 \mathrm{D}$, all of the resulting 21 homogenized elasticity parameters are very likely to be independent, which corresponds to triclinic anisotropy.

The forward modeling in the original model and the homogenized model uses a Ricker wavelet with $7.5 \mathrm{~Hz}$ central frequency as the source, which is located at the center of the model. Given the qP- and qSV-wave velocities that we calculate above, for the coarsegrid model, this corresponds to about 12 grid cells per dominant qSwavelength (approximately $252 \mathrm{~m}$ ) and 26 grid cells per dominant qP-wavelength (approximately $533 \mathrm{~m}$ ). Because each coarse grid contains $20 \times 20$ fine grids, these ratios are 20 times larger for the fine-grid model. We choose this central frequency to ensure that the error caused by numerical dispersion in the finite-difference modeling is as small as possible. By choosing these parameters, we also know that the dominant wavelength is approximately 25 times the size of the fine-grid heterogeneities for the qS-wave and approximately 50 times for the $\mathrm{qP}$-wave, assuming the average size of the heterogeneities shown in Figure $2 \mathrm{a}-2 \mathrm{f}$ is approximately $10 \mathrm{~m}$.

Figure 3a-3d compares the computed wavefield snapshots at $0.5 \mathrm{~s}$ from the two models. We see that the wavefields in the effective medium can accurately approximate the wavefields in the finegrid model. Furthermore, we compare directly the two wavefields at this same time at a depth of $2400 \mathrm{~m}$ in the model. We plot the fineand coarse-grid wavefields as black curve and dots, respectively, showing that they are in good agreement (Figure $4 \mathrm{a}$ and $4 \mathrm{~b}$ ). Because of the small grid size of the fine model, the modeling time 
a)

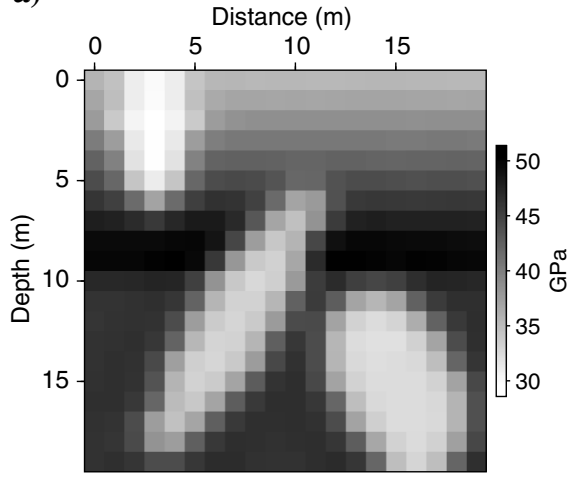

b)

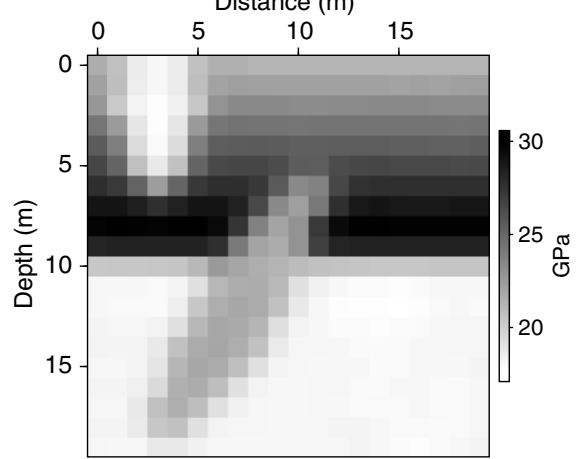

c)

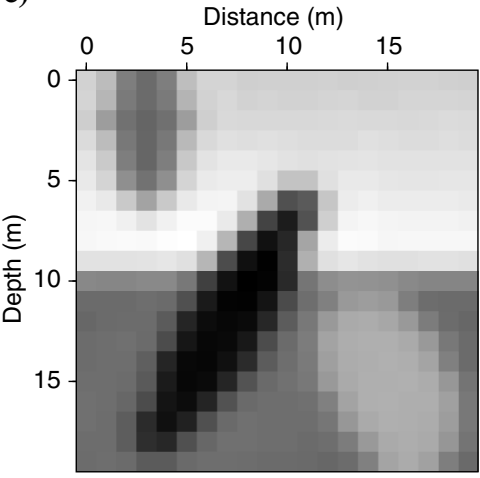

d)

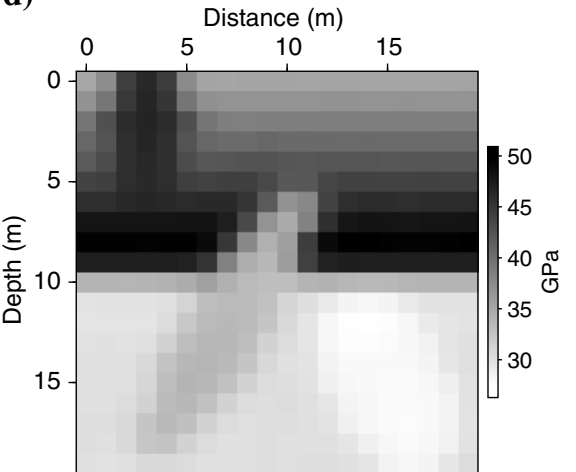

e)

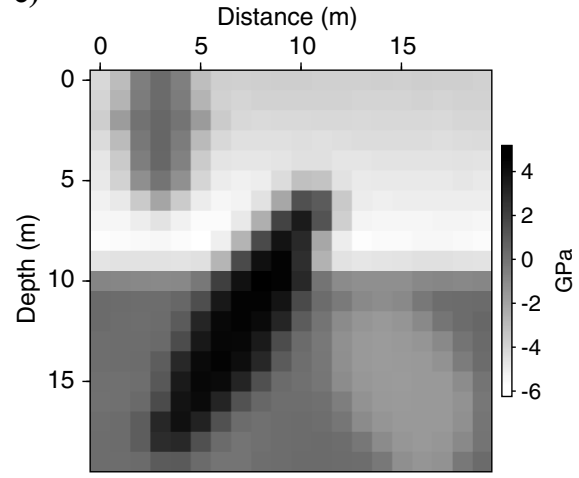

f)

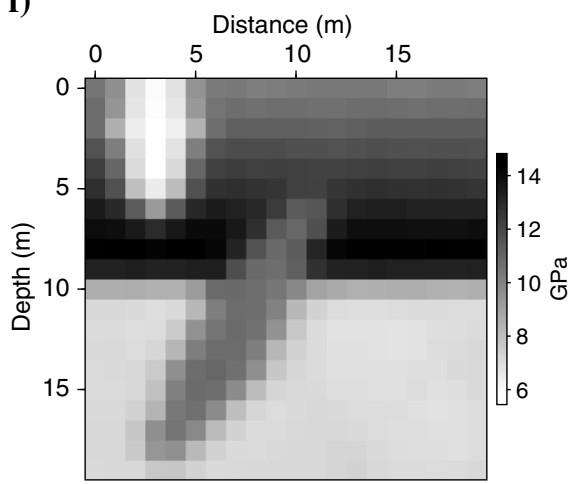

Figure 2. Subgrid elasticity parameter models. Panels (a-f) represent $C_{11}, C_{13}, C_{15}, C_{33}, C_{35}$, and $C_{55}$, respectively. The grid size is $1 \times 1 \mathrm{~m}$.

Figure 3. Comparisons of wavefields in the finegrid and effective media. Panels (a and $\mathrm{b}$ ) are $v_{1}$ and $v_{3}$ wavefields from the fine-grid model, respectively, and panels (c and d) are $v_{1}$ and $v_{3}$ wavefields from the effective model, respectively. a)

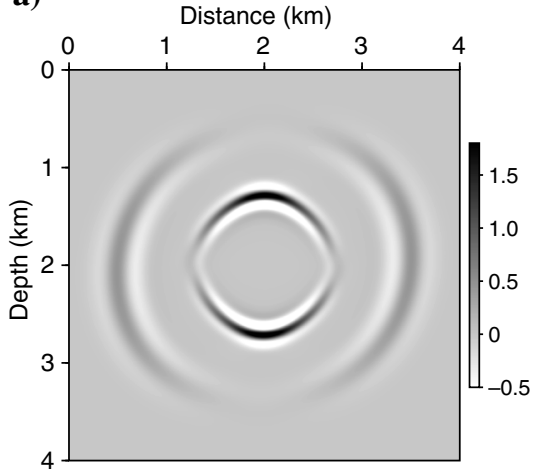

b)

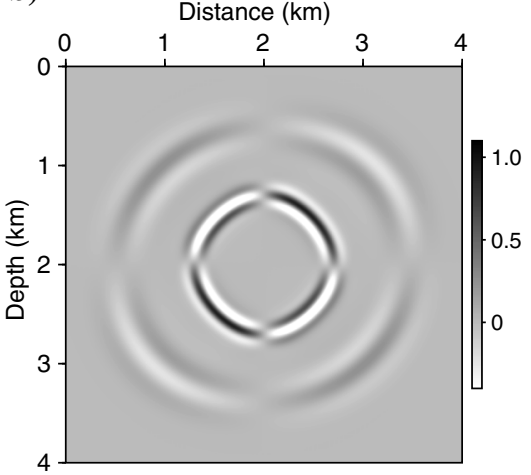

c)

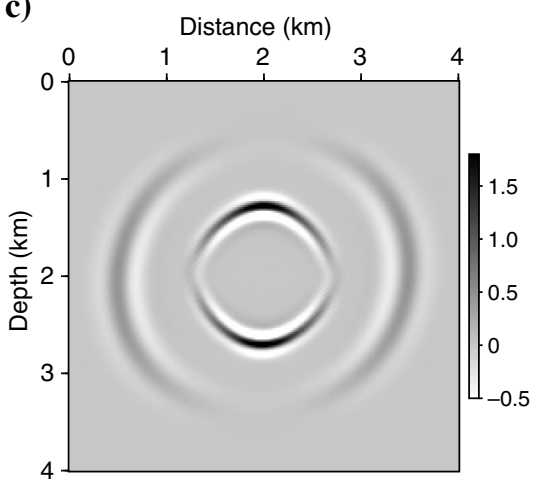

d)

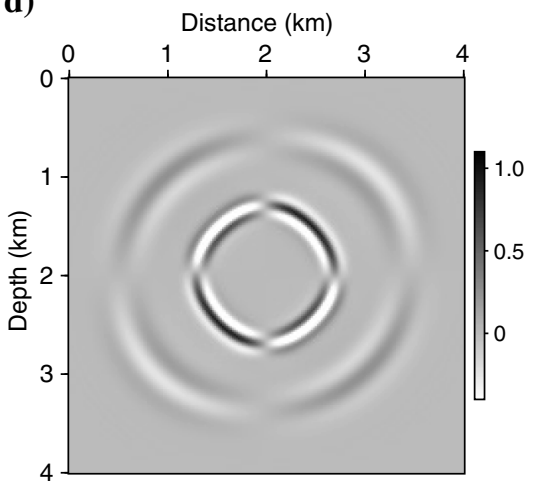


a)
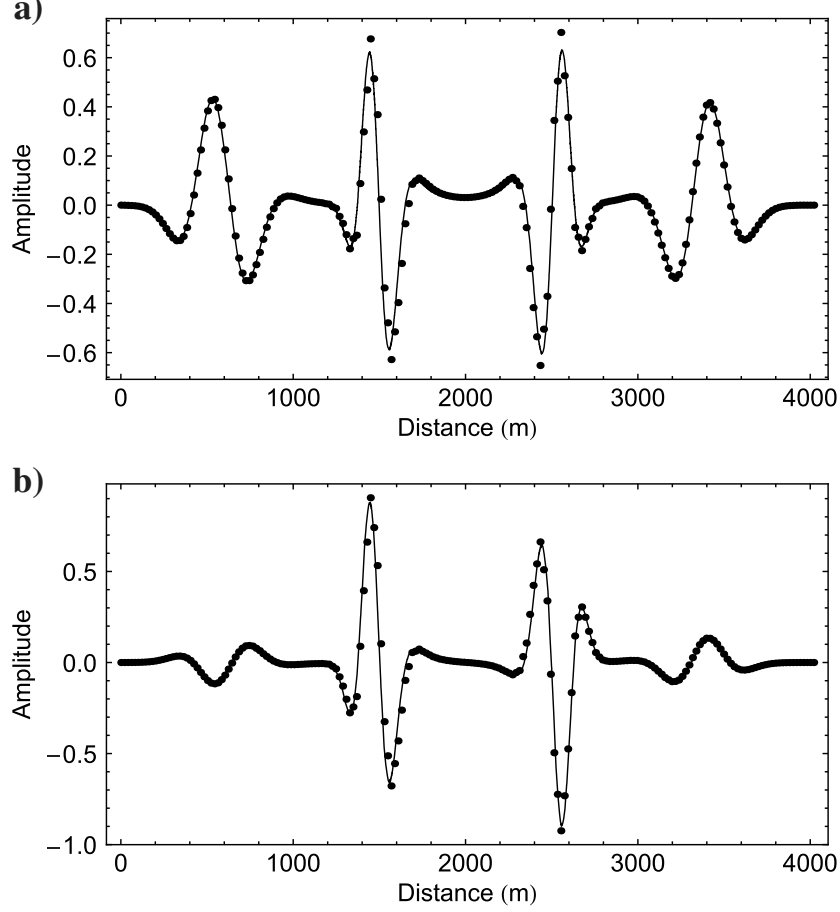

Figure 4. Comparison of (a) $v_{1}$ and (b) $v_{3}$ wavefields along a horizontal line at a depth of $2400 \mathrm{~m}$ of the snapshots in Figure 3. The black lines are the fine-grid solution, and the black dots are solutions from the effective medium. step has to be quite small to ensure stability, $0.1 \mathrm{~ms}$ in our modeling, whereas for the effective medium, we can safely use $1.0 \mathrm{~ms}$. This makes the ratio of computation time for the fine-grid homogenized medium simulations to be approximately $(93303 \mathrm{~s}) /(9.13 \mathrm{~s})$, i.e., $10^{4}$, for the same total wave propagation time $(0.5 \mathrm{~s})$.

The second model is a random medium generated with the von Kármán correlation function (Goff and Jordan, 1988; Klimeš, 2002), with correlation lengths of $30 \mathrm{~m}$ in the horizontal direction and $5 \mathrm{~m}$ in the vertical direction, respectively, and there are three horizontal reflectors within the model. The original model contains $2000 \times 2000$ grids, each $1 \times 1 \mathrm{~m}$ in size. Figure $5 \mathrm{a}-5 \mathrm{f}$ shows the elasticity constants in this model. We assume constant density of $1000 \mathrm{~kg} / \mathrm{m}^{3}$ for convenience. As in the previous example, such a model does not satisfy the basic assumptions of Backus averaging or the Schoenberg-Muir theory. We compute homogenized moduli and density values using a coarse grid with $200 \times 200$ cells, each of which is $10 \times 10 \mathrm{~m}$; i.e., each coarse grid contains $10 \times 10$ fine cells from the original model. The source placed at $(1000$ and $40 \mathrm{~m})$ is a Ricker wavelet with a central frequency of $15 \mathrm{~Hz}$. Because the elasticity parameters correspond to phase velocities from approximately 1350 to $2850 \mathrm{~m} / \mathrm{s}$ for the qSV-wave and approximately 2250 to $4800 \mathrm{~m} / \mathrm{s}$ for the $\mathrm{qP}$-wave, the dominant wavelength ranges from approximately 90 to $190 \mathrm{~m}$ for the qSV-wave and approximately 150 to $320 \mathrm{~m}$ for the qP-wave. This also implies that the dominant wavelength of the qSV-wave is at least 9 times the size of the coarse block we use for the numerical homogenization, and at least 15 times this size for the $\mathrm{qP}$-wave. a)

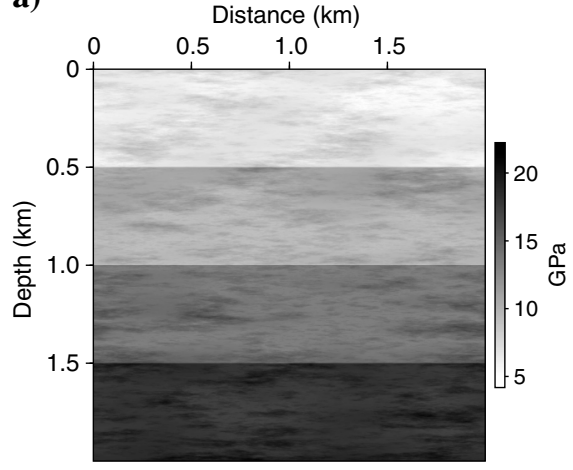

b)

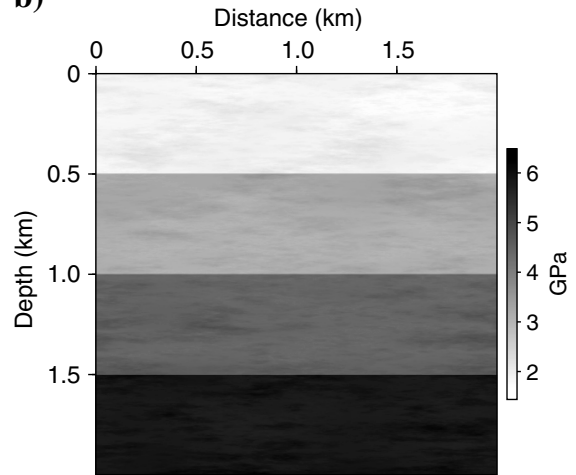

c)

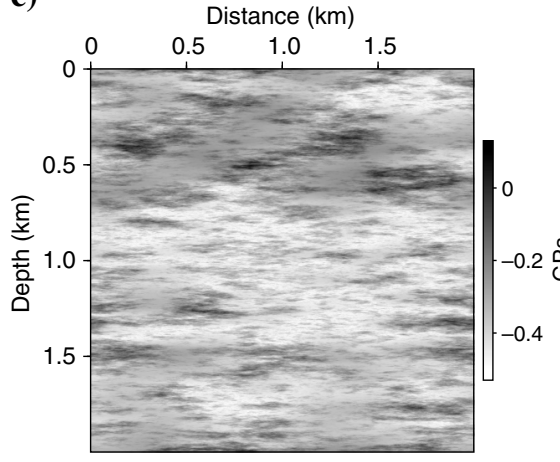

d)

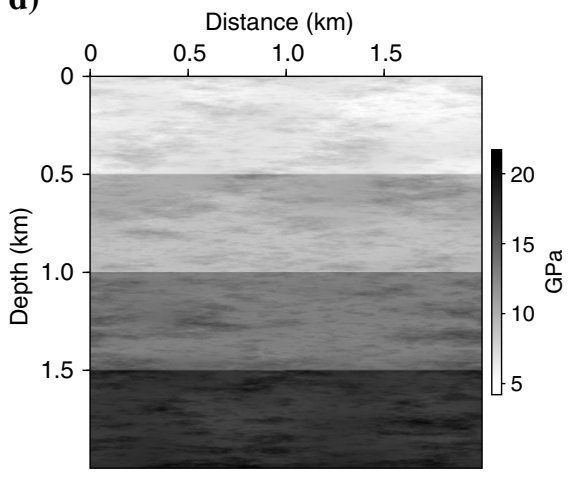

e)

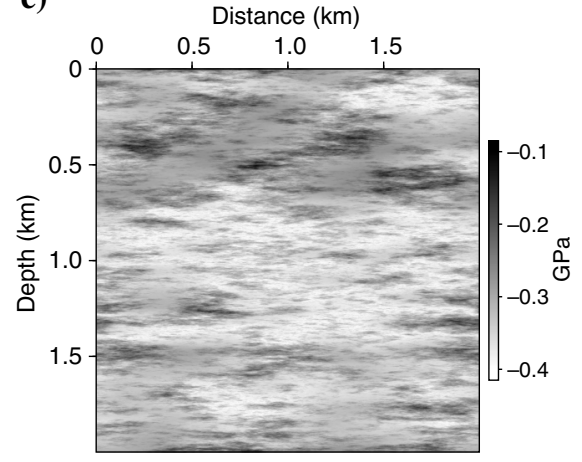

f)

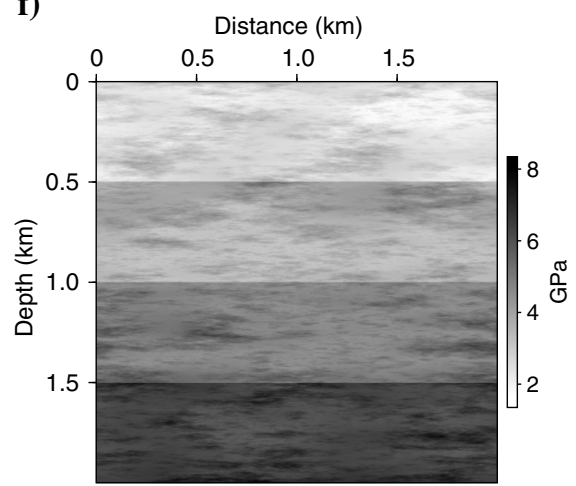

Figure 5. Four-layer von Kármán correlation random medium model. Panels (a-f) represent $C_{11}, C_{13}, C_{15}, C_{33}, C_{35}$, and $C_{55}$, respectively. The model contains $2000 \times 2000$ grids, each $1 \times 1 \mathrm{~m}$ in size. 
The receivers are at a depth of $40 \mathrm{~m}$, with horizontal coordinates ranging from 0 to $2000 \mathrm{~m}$ with an interval of $10 \mathrm{~m}$. We compare the wavefield snapshots and the seismograms calculated using the 20thorder RSG. Figure 6a-6d is the wavefield snapshots of $v_{1}$ and $v_{3}$ components at $0.4 \mathrm{~s}$. We again take a depth slice at $400 \mathrm{~m}$ from the wavefield snapshots and plot the fine- and coarse-grid wavefields as black curve and dots, respectively and they are in good agreement (Figure $7 \mathrm{a}$ and $7 \mathrm{~b}$ ). This visual comparison shows that the wavefields in our homogenized medium can well approximate the wavefield in the original random medium. Furthermore, Figures 8 and 9 show the seismograms that are clipped to display the reflections and scattered wavefields from the reflectors and heterogeneities in the model. The fine-grid model solution is plotted as blue wiggles, and our homogenized medium solution is plotted as red wiggles. There are only some inconsistencies in the seismogram at approximately $0.7 \mathrm{~s}$ in $v_{1}$ seismogram and at approximately $0.4-0.5 \mathrm{~s}$ in $v_{3}$ seismogram. For the other parts, our homogenized medium solution has very good consistency with the reference solution and can be considered as a satisfactory approximation of the original solution.

\section{Fractured medium}

We further verify our method by investigating a fractured medium. Sizes of natural fractures in rocks may range from several centimeters to hundreds of meters (Hobday and Worthington, 2012), and their alignment with preferred orientation in space may bring anisotropy (Tsvankin, 2005). In our test, we set up a fine-grid model that is composed of $60 \times 50$ coarse grids, and each coarse grid contains approximately 300 vertically oriented fractures that are randomly distributed in space as shown in Figure 10. Each coarse grid is discretized into $200 \times 200$ fine grids, leading to a total of $12,000 \times 10,000$ fine grids in the finely discretized model. The size of the fine grid is $0.01 \times 0.01 \mathrm{~m}$. The width of each fracture is 1 fine grid, i.e., $1 \mathrm{~cm}$, and the length is 10 fine grids, or $10 \mathrm{~cm}$. We assume that the fractures are filled with a soft, isotropic medium
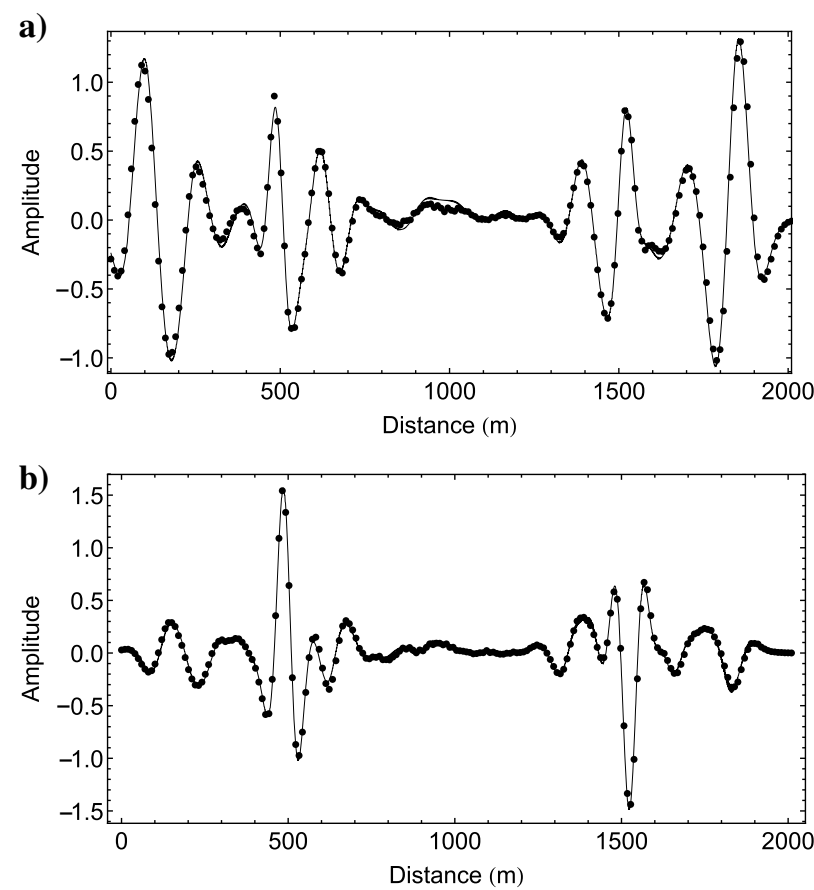

Figure 7. Comparison of (a) $v_{1}$ and (b) $v_{3}$ wavefields along a horizontal line at a depth of $400 \mathrm{~m}$ of the snapshots in Figure 6. The black lines are the fine-grid solution, and the black dots are solutions from our homogenized medium.
Figure 6. Comparisons of wavefield snapshots at $0.4 \mathrm{~s}$. Panels ( $\mathrm{a}$ and $\mathrm{b}$ ) are $v_{1}$ and $v_{3}$ wavefields from the fine-grid model, respectively, and panels (c) and (d) are $v_{1}$ and $v_{3}$ wavefields from our homogenized parameter model, respectively.
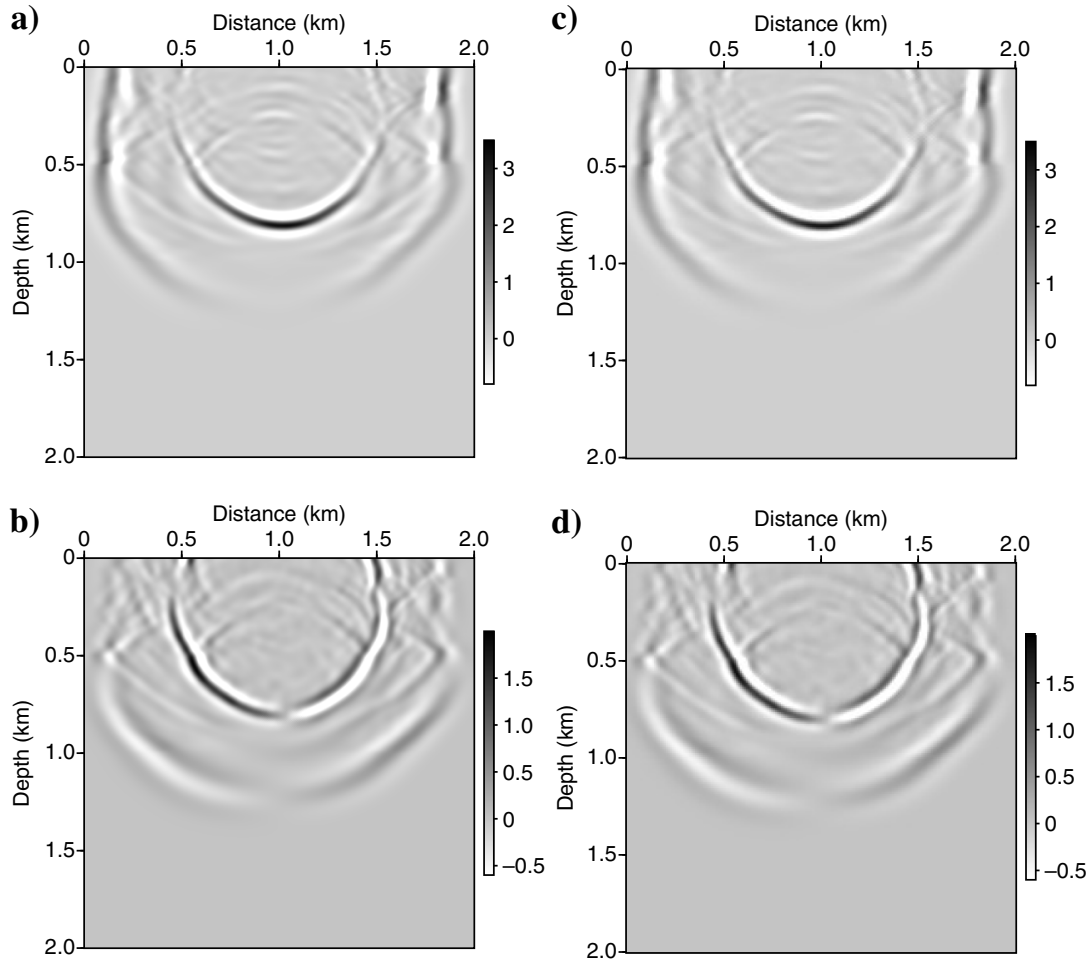

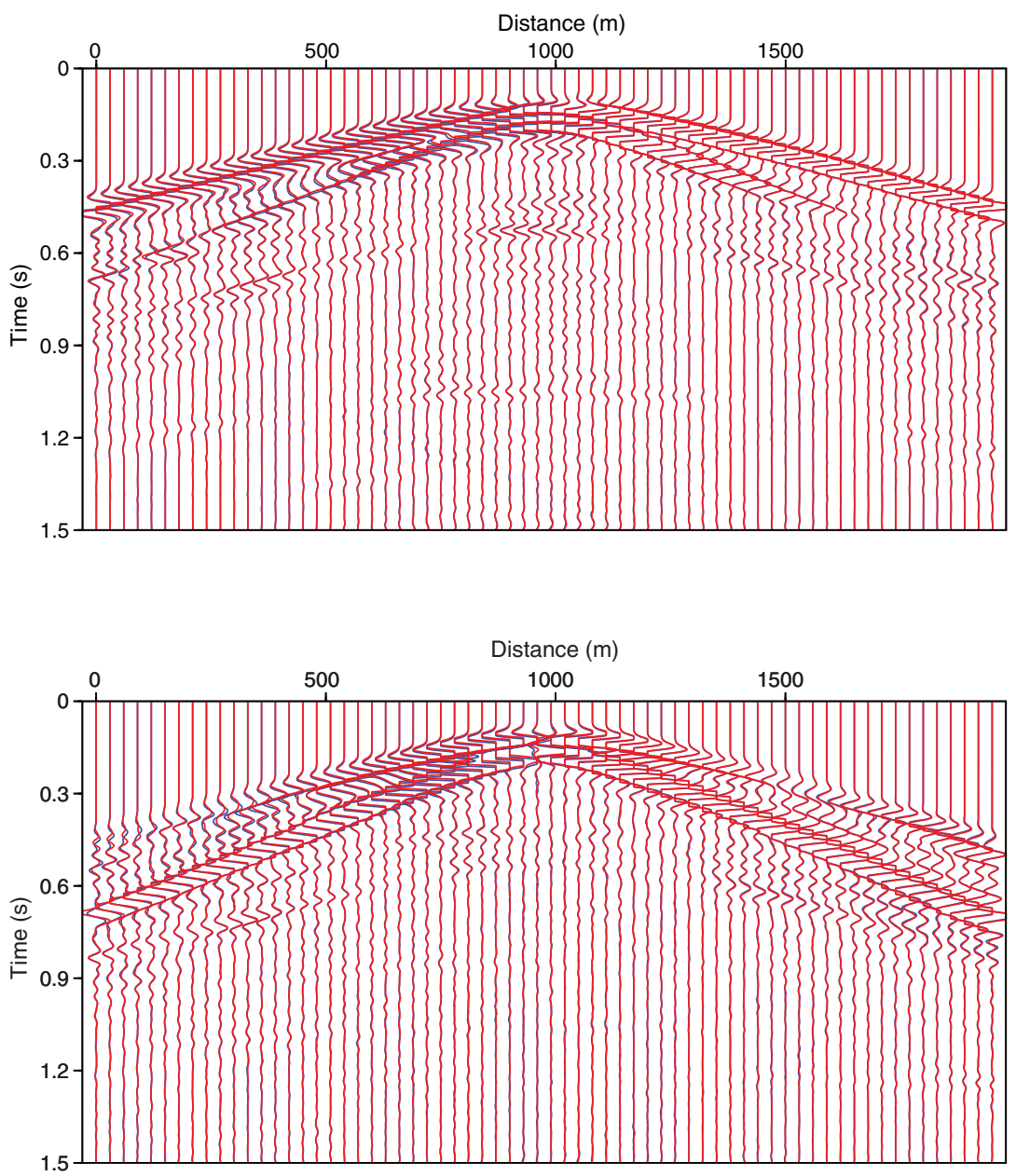

Figure 8. Comparisons of seismograms showing $v_{1}$ components for the original fine-grid solution (blue) and the solution with homogenized parameters (red). We clip the seismograms so that the waveforms of reflections and scattered fields have amplitudes large enough to be seen.

Figure 9. Comparisons of seismograms of showing $v_{3}$ component for the original fine-grid solution (blue) and the solution with homogenized parameters (red). The seismograms are clipped to exaggerate reflected and scattered waves as in Figure 8. with $C_{11}=0.667 \mathrm{GPa}$ and $C_{55}=0.357 \mathrm{GPa}$. The model has an isotropic, homogeneous background with $C_{11}=9.0 \mathrm{GPa}$ and $C_{55}=4.0 \mathrm{GPa}$. We again solve the elastic wave equation numerically with the RSG finite-difference method on the fine-grid model. Considering the size and medium properties of the fine grid, we use a Ricker wavelet with $f_{0}=250 \mathrm{~Hz}$ central frequency as the source, which is placed at the center $(50$ and $60 \mathrm{~m})$ of the model. The homogenized effective medium parameters from our multiscale basis function method are

$$
\mathbf{C}_{\text {multiscale }}=\left(\begin{array}{ccc}
3.6687 & 0.2862 & 0 \\
& 6.0283 & 0 \\
& & 2.0312
\end{array}\right) \mathrm{GPa} \text {, }
$$

with homogeneous density of $\rho=1000 \mathrm{~kg} / \mathrm{m}^{3}$. This indicates that the homogenized effective medium is TI with HTI. The group velocity along the $x_{1}$-axis is approximately 1915 and $2455 \mathrm{~m} / \mathrm{s}$ along the $x_{3}$-axis, and the Thomsen parameters (Thomsen, 1986) are $\epsilon \approx 0.322$ and $\delta \approx-0.030$. Such anisotropy is consistent with our expectation because the effective compliance theory for fractured rocks (e.g., Sayers and Kachanov, 1995; Sayers, 2002, 2006) tells us that a set of vertically aligned fractures is likely to result in HTI anisotropy. We then compare the results from finite-difference method with our homogenized solution. Figure 11a

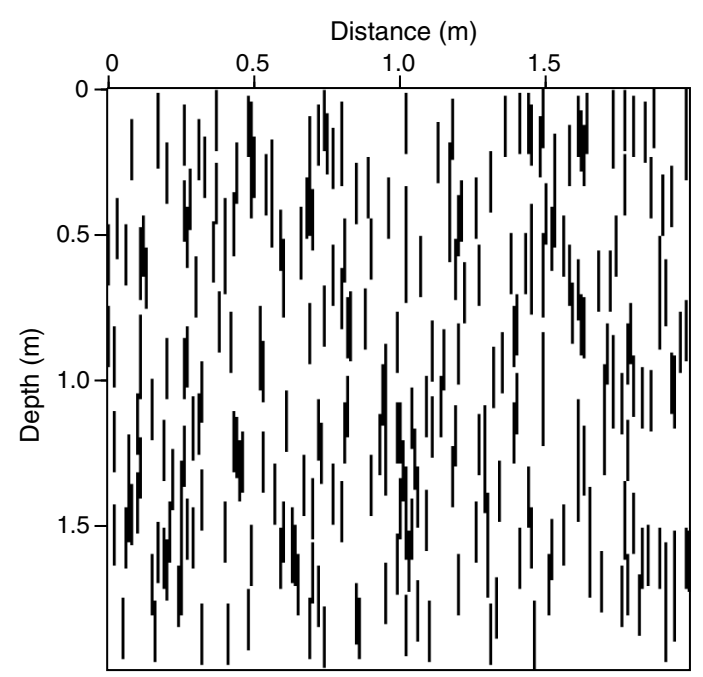

Figure 10. A coarse-grid model that is discretized with $200 \times 200$ fine grids. The fine grid size is $1 \mathrm{~cm}$ in both directions. The black lines represent randomly distributed vertical fractures, with width of 1 fine grid $(1 \mathrm{~cm})$ and length of 10 fine grids $(10 \mathrm{~cm})$. Our fine-grid model is composed of $60 \times 50$ such coarse grids. 
and $11 \mathrm{~b}$ shows the wavefield snapshots of the $v_{1}$ and $v_{3}$ components at $0.0250 \mathrm{~s}$ after source excitation, respectively. We also calculated the group velocity curves of $\mathrm{qP}$ - and $\mathrm{qSV}$-waves based on our result in equation 38 with the method described in Carcione (2007). In Figure $11 \mathrm{a}$ and $11 \mathrm{~b}$, the blue dashed curve is the group velocity curve for the $\mathrm{qP}$-wave and red is for the $\mathrm{qSV}$-wave. We remark that in the modeling, the source wavelet is shifted by $1.25 / f_{0}=0.005 \mathrm{~s}$ in time to make sure that the wavelet is almost zero at $t=0$, and therefore, the group velocity curves are calculated at $t=0.02 \mathrm{~s}$, so that they represent the position of peak amplitude of $\mathrm{qP}$ - and qSVwavefronts. It is obvious that these curves are in good consistency with the finite-difference modeling results on the fine grid, except

a)

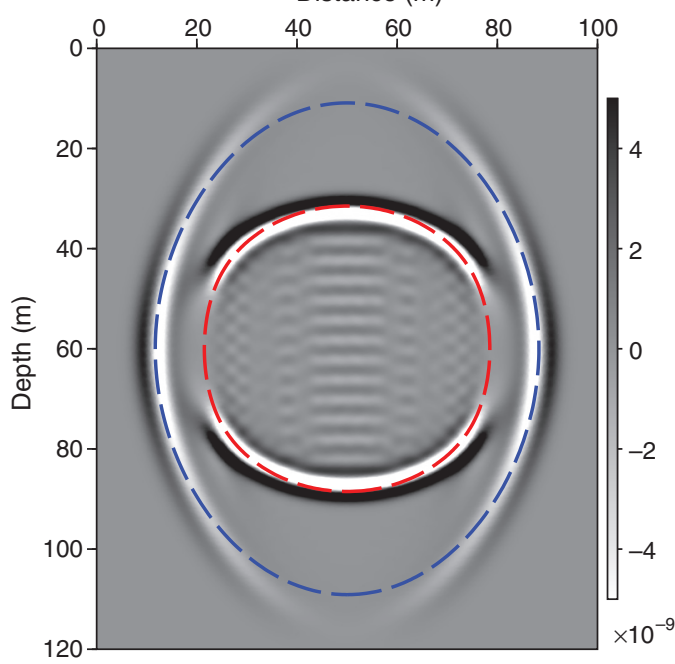

b)

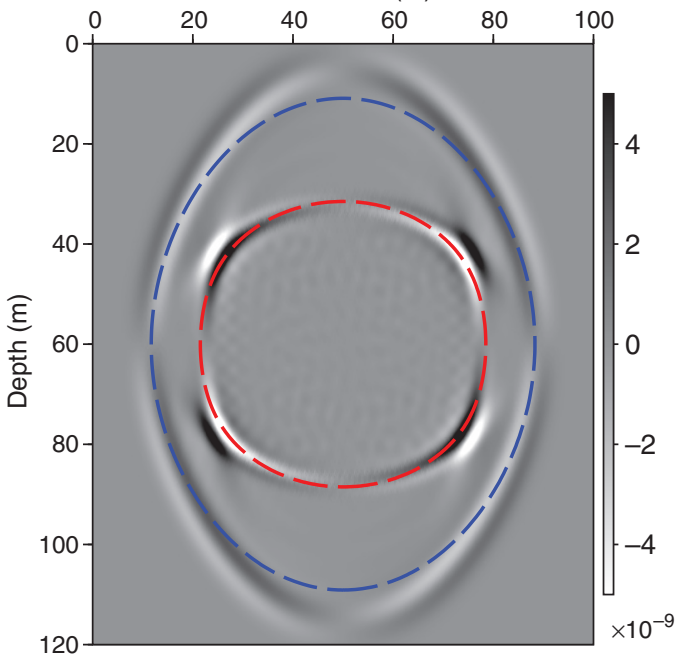

Figure 11. Comparison between the wavefield solutions on the fine mesh calculated with the RSG finite-difference method (the grayscale plots) and the group velocity curves predicted based on the homogenized effective medium parameters from our numerical homogenization method (the blue and red dashed curves). Panel (a) is the $v_{1}$ component wavefield snapshot whereas panel (b) is the $v_{3}$ component snapshot at $0.0250 \mathrm{~s}$. In panels (a and $\mathrm{b}$ ), the blue dashed curve represents the qP-wave group velocity and the red dashed curve represents the qSV-wave group velocity. that the predicted $\mathrm{qP}$-wave group velocity along $x_{3}$-axis is a little slower than the peak amplitude position in the finite-difference modeling results. This might result from inaccuracies when solving the local problems with the second-order FEM because there are large property contrasts between the background medium and the fracture. This consistency demonstrates the accuracy of our calculated homogenized effective parameters. Note that in this example, the dominant wavelength is approximately $10 \mathrm{~m}$, which is about 100 times of the fracture's length. This test shows that our multiscale method can give a good estimation of the elastic properties of the fractured medium with fracture sizes on the order of centimeters.

\section{CONCLUSIONS}

We have provided a numerical homogenization method for anisotropic elastic media. This method is constructed based on the multiscale theory previously developed for reducing the computation cost of wave equation modeling. Specifically, we have defined a local linear elasticity problem with appropriate boundary conditions, from which we can obtain multiscale basis functions for stress components. The coefficients for the finite-difference-like terms based on these multiscale basis functions can be considered as the numerical homogenized effective medium parameters. Our method is applicable to calculate the homogenized medium parameters for arbitrary subgrid medium property variations in which the ratio between the dominant wavelength and the average size of the arbitrary heterogeneities ranges from 10 to 100 . We have used several numerical examples, including horizontally layered media, media with arbitrarily heterogeneous subgrid variations, as well as a fractured medium with vertically aligned fractures, to demonstrate the effectiveness of our method, and we found that our method could give a good estimation of the effective medium parameters for anisotropic, heterogeneous media.

\section{ACKNOWLEDGMENTS}

The project is partially supported by Saudi Aramco, and partially by the U.S. Department of Energy under grant no. DE-FG0300ER15034. E. Chung's research is partially supported by the Hong Kong RGC General Research Fund (project no. 400411) and the CUHK Faculty of Science Research Incentive Fund. We appreciate editor E. Slob, assistant editor J. Shragge, associate editor E. Saenger, and the four anonymous reviewers for their valuable comments and suggestions that have greatly improved the quality of the manuscript.

\section{APPENDIX A}

\section{LOCAL STATIC LINEAR ELASTICITY PROBLEM SOLVED WITH SECOND-ORDER FINITE-ELEMENT METHOD}

The local problem in equation 6 with boundary conditions 7 can be solved with the standard continuous Galerkin FEM (e.g., Larson and Bengzon, 2013). Here, we present some details to illustrate the procedure.

First, we transform equation 6 into the form that is more commonly used in FEM with fourth-order elasticity tensor $\mathbf{c}=\mathbf{c}_{i j k l}$ : 


$$
-\nabla \cdot \boldsymbol{\sigma}=\mathbf{0}, \quad \boldsymbol{\sigma}=\mathbf{c}: \boldsymbol{\varepsilon}, \quad \boldsymbol{\varepsilon}=\frac{1}{2}\left[\nabla \mathbf{u}+(\nabla \mathbf{u})^{\mathrm{T}}\right]
$$

where $\varepsilon$ is the strain tensor. For the boundary conditions 7, it is easy to show that they are equivalent to the following Neumann boundary conditions:

$$
\begin{aligned}
& \boldsymbol{\sigma} \cdot \mathbf{n}_{1}^{+}=\left(\sigma_{11}, \sigma_{13}\right)=(1,1), \\
& \boldsymbol{\sigma} \cdot \mathbf{n}_{1}^{-}=\left(-\sigma_{11},-\sigma_{13}\right)=(-1,-1), \\
& \boldsymbol{\sigma} \cdot \mathbf{n}_{3}^{+}=\left(\sigma_{13}, \sigma_{33}\right)=(1,1), \text { and } \\
& \boldsymbol{\sigma} \cdot \mathbf{n}_{3}^{-}=\left(-\sigma_{13},-\sigma_{33}\right)=(-1,-1),
\end{aligned}
$$

where $\mathbf{n}_{1}^{+}=(1,0)$ is the outward-pointed normal of the right vertical boundary, $\mathbf{n}_{1}^{-}=(-1,0)$ is the outward-pointed normal of the left vertical boundary, $\mathbf{n}_{3}^{+}=(0,1)$ is the outward-pointed normal of the bottom horizontal boundary, and $\mathbf{n}_{3}^{-}=(0,-1)$ is the outwardpointed normal of the top horizontal boundary.

The elasticity problem must be solved in each of the four subrectangles within the support $K_{\sigma}$ for the stress variables (Figure 1), and we assume that each subrectangle is composed of $r_{1} r_{3}$ finer elements. The elastic parameters $\mathbf{c}$ are homogeneous on a fine element but generally heterogeneous within the rectangle; the discretization is shown in Figure A-1.

The above problem and discretization results in the following weak form:

$$
\int_{K} \boldsymbol{\sigma}(\mathbf{u}): \boldsymbol{\varepsilon}(\mathbf{u}) \mathrm{d} \mathbf{x}=\int_{\partial K} \boldsymbol{\sigma}(\mathbf{u}) \cdot \mathbf{n} \mathrm{d} s,
$$

with $\mathbf{n}$ being the outward pointed normal on each edge of $K$. We further have the discrete form:

$$
\mathbf{A} \mathbf{U}=\mathbf{B}
$$

where we assemble $\mathbf{A}$ and $\mathbf{B}$ with second-order finite-element basis functions (e.g., Larson and Bengzon, 2013). Given the analytic expressions for the basis functions and the discretization in Figure A-1, we then develop exact expressions for $\mathbf{A}$ and $\mathbf{B}$ for a fine element $K_{h}$ using Mathematica to complete the algebra:

$$
\mathbf{A}_{K_{h}}=\left(\begin{array}{cccccccc}
A_{11} & A_{12} & A_{13} & A_{14} & A_{15} & A_{16} & A_{17} & A_{18} \\
& A_{22} & A_{23} & A_{24} & A_{25} & A_{26} & A_{27} & A_{28} \\
& & A_{33} & A_{34} & A_{35} & A_{36} & A_{37} & A_{38} \\
& & & A_{44} & A_{45} & A_{46} & A_{47} & A_{48} \\
& & & & A_{55} & A_{56} & A_{57} & A_{58} \\
& & & & & A_{66} & A_{67} & A_{68} \\
& & & & & & A_{77} & A_{78} \\
& & & & & & & A_{88}
\end{array}\right),
$$

where

$$
A_{11}=\frac{1}{6}\left(\left(2 C_{11} \Delta x_{3}\right) / \Delta x_{1}+3 C_{15}+\left(2 C_{55} \Delta x_{1}\right) / \Delta x_{3}\right),
$$

$$
\begin{aligned}
A_{12}= & \frac{1}{12}\left(3\left(C_{13}+C_{55}\right)+\left(4 C_{35} \Delta x_{1}\right) / \Delta x_{3}\right. \\
& \left.+\left(4 C_{15} \Delta x_{3}\right) / \Delta x_{1}\right), \\
A_{13}= & \left(C_{55} \Delta x_{1}\right) /\left(6 \Delta x_{3}\right)-\left(C_{11} \Delta x_{3}\right) /\left(3 \Delta x_{1}\right), \\
A_{14}= & \frac{1}{12}\left(3 C_{13}-3 C_{55}+\left(2 C_{35} \Delta x_{1}\right) / \Delta x_{3}\right. \\
& \left.-\left(4 C_{15} \Delta x_{3}\right) / \Delta x_{1}\right),
\end{aligned}
$$

$$
A_{15}=\frac{1}{6}\left(-3 C_{15}-\left(C_{55} \Delta x_{1}\right) / \Delta x_{3}-\left(C_{11} \Delta x_{3}\right) / \Delta x_{1}\right) \text {, }
$$

$$
\begin{aligned}
A_{16}= & \frac{1}{12}\left(-3\left(C_{13}+C_{55}\right)-\left(2 C_{35} \Delta x_{1}\right) / \Delta x_{3}\right. \\
& \left.-\left(2 C_{15} \Delta x_{3}\right) / \Delta x_{1}\right),
\end{aligned}
$$

$$
A_{17}=-\left(\left(C_{55} \Delta x_{1}\right) /\left(3 \Delta x_{3}\right)\right)+\left(C_{11} \Delta x_{3}\right) /\left(6 \Delta x_{1}\right),
$$

$$
\begin{aligned}
A_{18}= & \frac{1}{12}\left(-3 C_{13}+3 C_{55}-\left(4 C_{35} \Delta x_{1}\right) / \Delta x_{3}\right. \\
& \left.+\left(2 C_{15} \Delta x_{3}\right) / \Delta x_{1}\right),
\end{aligned}
$$

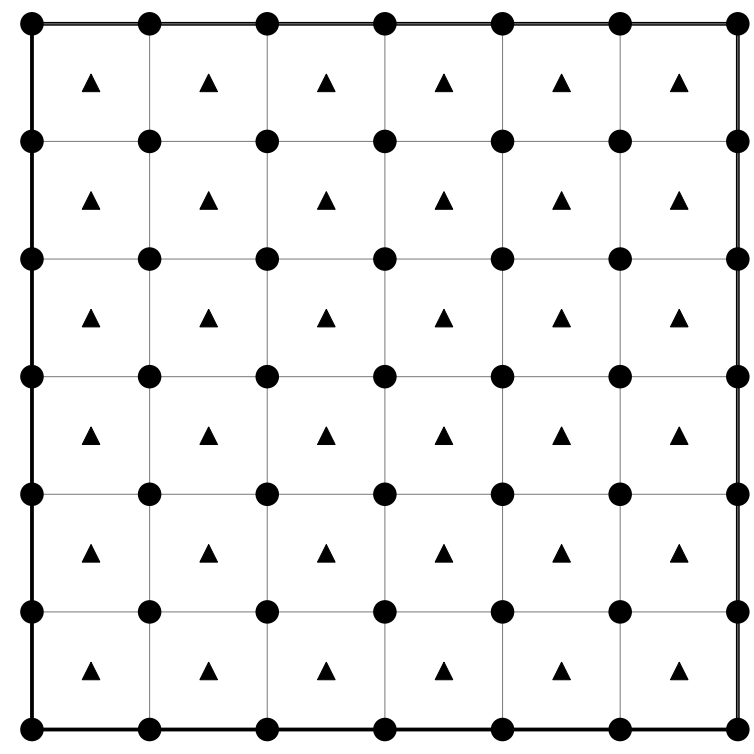

Figure A-1. A sketch of the fine mesh discretization for the local problem. To solve the local problem with the FEM, we compute displacement $\mathbf{u}$ at the locations $\bullet$, and after the calculation of $\mathbf{u}$, we can obtain the stress for a fine element at the center of a fine element at locations $\mathbf{m}$. 


$$
\begin{aligned}
A_{22}= & \frac{1}{6}\left(3 C_{35}+\left(2 C_{33} \Delta x_{1}\right) / \Delta x_{3}+\left(2 C_{55} \Delta x_{3}\right) / \Delta x_{1}\right), \\
A_{23}= & \frac{1}{12}\left(-3 C_{13}+3 C_{55}+\left(2 C_{35} \Delta x_{1}\right) / \Delta x_{3}\right. \\
& \left.-\left(4 C_{15} \Delta x_{3}\right) / \Delta x_{1}\right), \\
A_{24}= & \left(C_{33} \Delta x_{1}\right) /\left(6 \Delta x_{3}\right)-\left(C_{55} \Delta x_{3}\right) /\left(3 \Delta x_{1}\right), \\
A_{25}= & \frac{1}{12}\left(-3\left(C_{13}+C_{55}\right)-\left(2 C_{35} \Delta x_{1}\right) / \Delta x_{3}\right. \\
& \left.-\left(2 C_{15} \Delta x_{3}\right) / \Delta x_{1}\right), \\
A_{26}= & \frac{1}{6}\left(-3 C_{35}-\left(C_{33} \Delta x_{1}\right) / \Delta x_{3}-\left(C_{55} \Delta x_{3}\right) / \Delta x_{1}\right), \\
A_{27}= & \frac{1}{12}\left(3 C_{13}-3 C_{55}-\left(4 C_{35} \Delta x_{1}\right) / \Delta x_{3}\right. \\
& \left.+\left(2 C_{15} \Delta x_{3}\right) / \Delta x_{1}\right),
\end{aligned}
$$

$$
A_{44}=\frac{1}{6}\left(-3 C_{35}+\left(2 C_{33} \Delta x_{1}\right) / \Delta x_{3}+\left(2 C_{55} \Delta x_{3}\right) / \Delta x_{1}\right),
$$

$$
\begin{aligned}
A_{45}= & \frac{1}{12}\left(-3 C_{13}+3 C_{55}-\left(4 C_{35} \Delta x_{1}\right) / \Delta x_{3}\right. \\
& \left.+\left(2 C_{15} \Delta x_{3}\right) / \Delta x_{1}\right)
\end{aligned}
$$

$$
A_{46}=-\left(\left(C_{33} \Delta x_{1}\right) /\left(3 \Delta x_{3}\right)\right)+\left(C_{55} \Delta x_{3}\right) /\left(6 \Delta x_{1}\right),
$$

$$
\begin{aligned}
A_{47}= & \frac{1}{12}\left(3\left(C_{13}+C_{55}\right)-\left(2 C_{35} \Delta x_{1}\right) / \Delta x_{3}\right. \\
& \left.-\left(2 C_{15} \Delta x_{3}\right) / \Delta x_{1}\right),
\end{aligned}
$$

$$
A_{48}=\frac{1}{6}\left(3 C_{35}-\left(C_{33} \Delta x_{1}\right) / \Delta x_{3}-\left(C_{55} \Delta x_{3}\right) / \Delta x_{1}\right),
$$

$$
A_{55}=\frac{1}{6}\left(3 C_{15}+\left(2 C_{55} \Delta x_{1}\right) / \Delta x_{3}+\left(2 C_{11} \Delta x_{3}\right) / \Delta x_{1}\right),
$$

$$
A_{33}=\frac{1}{6}\left(-3 C_{15}+\left(2 C_{55} \Delta x_{1}\right) / \Delta x_{3}+\left(2 C_{11} \Delta x_{3}\right) / \Delta x_{1}\right),
$$

$$
\begin{aligned}
A_{34}= & \frac{1}{12}\left(-3\left(C_{13}+C_{55}\right)+\left(4 C_{35} \Delta x_{1}\right) / \Delta x_{3}\right. \\
& \left.+\left(4 C_{15} \Delta x_{3}\right) / \Delta x_{1}\right)
\end{aligned}
$$

$A_{35}=-\left(\left(C_{55} \Delta x_{1}\right) /\left(3 \Delta x_{3}\right)\right)+\left(C_{11} \Delta x_{3}\right) /\left(6 \Delta x_{1}\right),(\mathrm{A}-23)$

$$
\begin{aligned}
A_{56}= & \frac{1}{12}\left(3\left(C_{13}+C_{55}\right)+\left(4 C_{35} \Delta x_{1}\right) / \Delta x_{3}\right. \\
& \left.+\left(4 C_{15} \Delta x_{3}\right) / \Delta x_{1}\right)
\end{aligned}
$$

$$
A_{57}=\left(C_{55} \Delta x_{1}\right) /\left(6 \Delta x_{3}\right)-\left(C_{11} \Delta x_{3}\right) /\left(3 \Delta x_{1}\right),
$$

$$
\begin{aligned}
A_{58}= & \frac{1}{12}\left(3 C_{13}-3 C_{55}+\left(2 C_{35} \Delta x_{1}\right) / \Delta x_{3}\right. \\
& \left.-\left(4 C_{15} \Delta x_{3}\right) / \Delta x_{1}\right)
\end{aligned}
$$

$$
\begin{aligned}
A_{36}= & \frac{1}{12}\left(3 C_{13}-3 C_{55}-\left(4 C_{35} \Delta x_{1}\right) / \Delta x_{3}\right. \\
& \left.+\left(2 C_{15} \Delta x_{3}\right) / \Delta x_{1}\right)
\end{aligned}
$$

$$
A_{66}=\frac{1}{6}\left(3 C_{35}+\left(2 C_{33} \Delta x_{1}\right) / \Delta x_{3}+\left(2 C_{55} \Delta x_{3}\right) / \Delta x_{1}\right),
$$

$$
A_{37}=\frac{1}{6}\left(3 C_{15}-\left(C_{55} \Delta x_{1}\right) / \Delta x_{3}-\left(C_{11} \Delta x_{3}\right) / \Delta x_{1}\right),
$$

$$
\begin{aligned}
A_{67}= & \frac{1}{12}\left(-3 C_{13}+3 C_{55}+\left(2 C_{35} \Delta x_{1}\right) / \Delta x_{3}\right. \\
& \left.-\left(4 C_{15} \Delta x_{3}\right) / \Delta x_{1}\right)
\end{aligned}
$$




$$
\begin{gathered}
A_{68}=\left(C_{33} \Delta x_{1}\right) /\left(6 \Delta x_{3}\right)-\left(C_{55} \Delta x_{3}\right) /\left(3 \Delta x_{1}\right), \\
A_{77}=\frac{1}{6}\left(-3 C_{15}+\left(2 C_{55} \Delta x_{1}\right) / \Delta x_{3}+\left(2 C_{11} \Delta x_{3}\right) / \Delta x_{1}\right),
\end{gathered}
$$

$$
\begin{aligned}
A_{78}= & \frac{1}{12}\left(-3\left(C_{13}+C_{55}\right)+\left(4 C_{35} \Delta x_{1}\right) / \Delta x_{3}\right. \\
& \left.+\left(4 C_{15} \Delta x_{3}\right) / \Delta x_{1}\right),
\end{aligned}
$$

and

$$
A_{88}=\frac{1}{6}\left(-3 C_{35}+\left(2 C_{33} \Delta x_{1}\right) / \Delta x_{3}+\left(2 C_{55} \Delta x_{3}\right) / \Delta x_{1}\right),
$$

with $\Delta x_{1}$ being the fine-element edge length in the $x_{1}$-direction; $\Delta x_{3}$ being the fine-element edge length in the $x_{3}$-direction; and $C_{i j}$ are elasticity constants of the element $K_{h}$, which is assumed to be constant within $K_{h}$ but generally heterogeneous in a coarse element. If using higher order finite elements, $C_{i j}$ should be interpolated with some appropriate interpolation rules. Note that we only show the upper triangle part of $\mathbf{A}$ in equation A-5, and $\mathbf{A}$ is a symmetric matrix. For matrix $\mathbf{B}$, which is related to the boundary conditions, we have the element matrix $\mathbf{B}_{K_{h}}$ for the fine element $K_{h}$ on left, right, top, and boundaries as

$$
\left.\mathbf{B}_{K_{h}}\right|_{\text {left boundary }}=-\frac{1}{2}\left(\Delta x_{3}, \Delta x_{3}, 0,0,0,0, \Delta x_{3}, \Delta x_{3}\right)^{\mathrm{T}},
$$

$$
\left.\mathbf{B}_{K_{h}}\right|_{\text {right boundary }}=\frac{1}{2}\left(0,0, \Delta x_{3}, \Delta x_{3}, \Delta x_{3}, \Delta x_{3}, 0,0\right)^{\mathrm{T}},
$$

$$
\left.\mathbf{B}_{K_{h}}\right|_{\text {top boundary }}=-\frac{1}{2}\left(\Delta x_{1}, \Delta x_{1}, \Delta x_{1}, \Delta x_{1}, 0,0,0,0\right)^{\mathrm{T}},
$$

$$
\left.\mathbf{B}_{K_{h}}\right|_{\text {bottom boundary }}=\frac{1}{2}\left(0,0,0,0, \Delta x_{1}, \Delta x_{1}, \Delta x_{1}, \Delta x_{1}\right)^{\mathrm{T}},
$$

which again are calculated with exact integration rules.

The above system has $2\left(r_{1}+1\right)\left(r_{3}+1\right)$ degrees of freedom for $\mathbf{u}$ (including $u_{1}$ and $u_{3}$ ). In Larson and Bengzon (2013), the stress tensors on triangular elements can be obtained with the gradients of u calculated using the MATLAB built-in partial differential equation (PDE) function pdegrad. Because we have used rectangular elements, we calculate the stress tensor at the center of each fine element with the definition of equation A-1 using the RSG finite-difference scheme (Saenger et al., 2000). For example, for $\sigma_{11}$ at the $\left(j_{1}, j_{3}\right)$ th fine element, we have

$$
\begin{aligned}
& \sigma_{11}\left(j_{1}, j_{3}\right) \\
& =C_{11}\left(j_{1}, j_{3}\right) \frac{1}{2 \Delta x_{1}}\left\{\left[u_{1}\left(j_{1}+\frac{1}{2}, j_{3}+\frac{1}{2}\right)-u_{1}\left(j_{1}-\frac{1}{2}, j_{3}-\frac{1}{2}\right)\right]\right. \\
& \left.+\left[u_{1}\left(j_{1}+\frac{1}{2}, j_{3}-\frac{1}{2}\right)-u_{1}\left(j_{1}-\frac{1}{2}, j_{3}+\frac{1}{2}\right)\right]\right\} \\
& +C_{13}\left(j_{1}, j_{3}\right) \frac{1}{2 \Delta x_{3}}\left\{\left[u_{3}\left(j_{1}+\frac{1}{2}, j_{3}+\frac{1}{2}\right)-u_{3}\left(j_{1}-\frac{1}{2}, j_{3}-\frac{1}{2}\right)\right] .\right. \\
& \left.+\left[u_{3}\left(j_{1}+\frac{1}{2}, j_{3}-\frac{1}{2}\right)-u_{3}\left(j_{1}-\frac{1}{2}, j_{3}+\frac{1}{2}\right)\right]\right\} \\
& +C_{15}\left(j_{1}, j_{3}\right) \frac{1}{2 \Delta x_{1}}\left\{\left[u_{3}\left(j_{1}+\frac{1}{2}, j_{3}+\frac{1}{2}\right)-u_{3}\left(j_{1}-\frac{1}{2}, j_{3}-\frac{1}{2}\right)\right] .\right. \\
& \left.+\left[u_{3}\left(j_{1}+\frac{1}{2}, j_{3}-\frac{1}{2}\right)-u_{3}\left(j_{1}-\frac{1}{2}, j_{3}+\frac{1}{2}\right)\right]\right\} \\
& +\left[c_{15}\left(j_{1}, j_{3}\right) \frac{1}{2 \Delta x_{3}}\left\{\left[j_{1}\left(j_{1}+\frac{1}{2}, j_{3}+\frac{1}{2}\right)-j_{3}-\frac{1}{2}\right)-u_{1}\left(j_{1}-\frac{1}{2}, j_{3}+\frac{1}{2}\right)\right]\right\},
\end{aligned}
$$

where $\Delta x_{1}$ and $\Delta x_{3}$ are the lengths of the vertical and horizontal edges of the fine element, respectively. This solution, along with the solutions in the other three blocks, is further taken as the multiscale basis function $\phi_{11}$ of stress component $\sigma_{11}$, as described in the text. Multiscale basis functions for the other stress components can be calculated in the same way.

\section{APPENDIX B}

\section{NUMERICAL HOMOGENIZATION FOR 3D HETEROGENEOUS, GENERALLY ANISOTROPIC MEDIA}

For 3D heterogeneous, anisotropic elastic media, we decompose the support of $\boldsymbol{\sigma}$ with eight cubic blocks, and in each of the blocks, we assume that a local linear elasticity problem similar to equation 6 is satisfied. We can define boundary conditions similar to those in the $2 \mathrm{D}$ case. If we denote the two faces of a cubic block that are perpendicular to the $x_{i}$-axis with $\mathcal{F}_{i}$, the boundary conditions for each stress component will be

$$
\begin{gathered}
\sigma_{11}=1 \quad \text { on } \mathcal{F}_{1}, \\
\sigma_{22}=1 \text { on } \mathcal{F}_{2}, \\
\sigma_{33}=1 \text { on } \mathcal{F}_{3}, \\
\sigma_{23}=1 \text { on } \mathcal{F}_{2} \text { and } \mathcal{F}_{3}, \\
\sigma_{13}=1 \text { on } \mathcal{F}_{1} \text { and } \mathcal{F}_{3}, \\
\sigma_{12}=1 \text { on } \mathcal{F}_{1} \text { and } \mathcal{F}_{2} .
\end{gathered}
$$


This local problem can also be solved with the FEM, which is similar to the approach in Appendix A. After solving the local problems in each of the eight cubic blocks, we join them together to form the basis functions of $\boldsymbol{\sigma}$ in $K_{\sigma}$.

With algebraic manipulations similar to those for the 2D case, we finally have the $3 \mathrm{D}$ effective compliance matrix as

$\tilde{\mathbf{S}}=\left(\begin{array}{llllll}S_{11} \phi_{11} \phi_{11} & S_{12} \phi_{22} \phi_{11} & S_{13} \phi_{33} \phi_{11} & S_{14} \phi_{23} \phi_{11} & S_{15} \phi_{13} \phi_{11} & S_{16} \phi_{12} \phi_{11} \\ S_{12} \phi_{11} \phi_{22} & S_{22} \phi_{22} \phi_{22} & S_{23} \phi_{33} \phi_{22} & S_{24} \phi_{23} \phi_{22} & S_{25} \phi_{13} \phi_{22} & S_{26} \phi_{12} \phi_{22} \\ S_{13} \phi_{11} \phi_{33} & S_{23} \phi_{22} \phi_{33} & S_{33} \phi_{33} \phi_{33} & S_{34} \phi_{23} \phi_{33} & S_{35} \phi_{13} \phi_{33} & S_{36} \phi_{12} \phi_{33} \\ S_{14} \phi_{11} \phi_{23} & S_{24} \phi_{22} \phi_{23} & S_{34} \phi_{33} \phi_{23} & S_{44} \phi_{23} \phi_{23} & S_{45} \phi_{13} \phi_{23} & S_{46} \phi_{12} \phi_{23} \\ S_{15} \phi_{11} \phi_{13} & S_{25} \phi_{22} \phi_{13} & S_{35} \phi_{33} \phi_{13} & S_{45} \phi_{23} \phi_{13} & S_{55} \phi_{13} \phi_{13} & S_{56} \phi_{12} \phi_{13} \\ S_{16} \phi_{11} \phi_{12} & S_{26} \phi_{22} \phi_{12} & S_{36} \phi_{33} \phi_{12} & S_{46} \phi_{23} \phi_{12} & S_{56} \phi_{13} \phi_{12} & S_{66} \phi_{12} \phi_{12}\end{array}\right)$,

where, for quadrilateral elements,

$$
\begin{aligned}
S_{i j} \phi_{s t} \phi_{p q}= & \frac{1}{n_{1} n_{2} n_{3}} \sum_{j_{1}=1}^{n_{1}} \sum_{j_{2}=1}^{n_{2}} \sum_{j_{3}=1}^{n_{3}} S_{i j}\left(j_{1}, j_{2}, j_{3}\right) \\
& \times \phi_{s t}\left(j_{1}, j_{2}, j_{3}\right) \phi_{p q}\left(j_{1}, j_{2}, j_{3}\right)
\end{aligned}
$$

is a summation over all fine elements within $K_{\sigma} ; n_{i}$ is the number of fine elements along the $i$ th axis, with $i=1,2,3$; and $\phi_{i j}$ are the multiscale basis functions solved from local problem for $\sigma_{i j}$. The effective elasticity matrix for a coarse block $K_{\sigma}$ is

$$
\tilde{\mathbf{C}}=\tilde{\mathbf{S}}^{-1}
$$

Finally, for the density, we have

$$
\tilde{\rho}=\frac{1}{n_{1} n_{2} n_{3}} \sum_{j_{1}=1}^{m_{1}} \sum_{j_{2}=1}^{n_{2}} \sum_{j_{3}=1}^{n_{3}} \rho\left(j_{1}, j_{2}, j_{3}\right) .
$$

\section{APPENDIX C}

\section{LTH-ORDER ROTATED STAGGERED-GRID FI- NITE-DIFFERENCE SCHEME}

Throughout the numerical experiments to compare the wavefield in fine-scale media and in the effective media, we solve the anisotropic wave equation using the high-order RSG finite-difference method. Because the RSG finite-difference method for wave equations has already been described in previous work (e.g., Saenger et al., 2000; Saenger and Bohlen, 2004), here we only provide some of the key results. In RSG, the derivatives of a field variable $u$ defined at integer position $\left(i_{1}, i_{3}\right)$ are expressed as the summation or difference of the derivatives along rotated axes (Saenger et al., 2000); i.e.,

$$
\begin{aligned}
& \partial_{1} u=\frac{\Delta r}{2 \Delta x_{1}}\left(D_{3} u+D_{1} u\right), \\
& \partial_{3} u=\frac{\Delta r}{2 \Delta x_{3}}\left(D_{3} u-D_{1} u\right),
\end{aligned}
$$

with the conventional staggered-grid derivatives $D_{1}$ and $D_{3}$ along rotated axes expressed as
Table C-1. Coefficients for 20th-order staggered-grid fintiedifference stencil.

\begin{tabular}{cc}
$m$ & $c_{m}$ \\
\hline 1 & $1.2418160 \mathrm{E}+00$ \\
2 & $-1.1289240 \mathrm{E}-01$ \\
3 & $2.7094169 \mathrm{E}-02$ \\
4 & $-7.4434527 \mathrm{E}-03$ \\
5 & $1.9297841 \mathrm{E}-03$ \\
6 & $-4.3061300 \mathrm{E}-04$ \\
7 & $7.7077173 \mathrm{E}-05$ \\
8 & $-1.0216503 \mathrm{E}-05$ \\
9 & $8.8378061 \mathrm{E}-07$ \\
10 & $-3.7237577 \mathrm{E}-08$ \\
\hline
\end{tabular}

$$
\begin{aligned}
D_{1}= & \frac{1}{\Delta r} \sum_{m=1}^{L} c_{m}\left[u\left(i_{1}+m^{-}, i_{3}-m^{-}\right)\right. \\
& \left.-u\left(i_{1}-m^{-}, i_{3}+m^{-}\right)\right] \\
D_{3}= & \frac{1}{\Delta r} \sum_{m=1}^{L} c_{m}\left[u\left(i_{1}+m^{-}, i_{3}+m^{-}\right)\right. \\
& \left.-u\left(i_{1}-m^{-}, i_{3}-m^{-}\right)\right]
\end{aligned}
$$

where $m^{-}=m-1 / 2, \Delta r=\sqrt{\Delta x_{1}^{2}+\Delta x_{3}^{2}}, L$ is half of the order of spatial accuracy, $i_{1}$ and $i_{3}$ are the integers along the $x_{1}$ - and $x_{3}$-axes, respectively, and the staggered-grid coefficients can be calculated as (Fornberg, 1990)

$$
c_{m}=\frac{(-1)^{L+1} \prod_{n=1, n \neq m}^{L}(2 n-1)^{2}}{(2 m-1) \prod_{n=1, n \neq m}^{L}\left[(2 m-1)^{2}-(2 n-1)^{2}\right]} .
$$

In our numerical tests, we use 20th-order spatial accuracy, i.e., $L=10$, to calculate the spatial differential operators in the anisotropic elastic wave equation, and the finite-difference coefficients are listed in Table C-1.

\section{REFERENCES}

Abdulle, A., and M. J. Grote, 2011, Finite element heterogeneous multiscale method for the wave equation: Multiscale Modeling and Simulation, 9 766-792, doi: 10.1137/100800488.

Backus, G. E., 1962, Long-wave elastic anisotropy produced by horizontal layering: Journal of Geophysical Research, 67, 4427-4440, doi: 10.1029/ JZ067i011p04427.

Budiansky, B., and R. J. O’Connell, 1976, Elastic moduli of a cracked solid International Journal of Solids and Structures, 12, 81-97, doi: 10.1016/ 0020-7683(76)90044-5.

Carcione, J., S. Picotti, F. Cavallini, and J. Santos, 2012, Numerical test of the Schoenberg-Muir theory: Geophysics, 77, no. 2, C27-C35, doi: 10 .1190/geo2011-0228.1.

Carcione, J. M., 2007, Wave fields in real media: Theory and numerical simulation of wave propagation in anisotropic, anelastic, porous and electromagnetic media 2nd ed.: Elsevier Science Handbook of Geophysical Exploration: Seismic Exploration, 38.

Chung, E., Y. Efendiev, and W. T. Leung, 2013, Generalized multiscale finite element methods for wave propagation in heterogeneous media: arXiv: 1307.0123

Chung, E. T., Y. Efendiev, and R. L. Gibson Jr., 2011b, Multiscale finite-element modeling of acoustic wave propagation: 81st Annual International meeting, SEG, Expanded Abstracts, 2898-2903. 
Chung, E. T., Y. Efendiev, and R. L. Gibson Jr., 2011a, An energyconserving discontinuous multiscale finite element method for the wave equation in heterogeneous media: Advances in Adaptive Data Analysis, $\mathbf{3}$ 251-268, doi: 10.1142/S1793536911000842.

Dablain, M., 1986, The application of high-order differencing to the scalar wave equation: Geophysics, 51, 54-66, doi: 10.1190/1.1442040.

Efendiev, Y., and T. Y. Hou, 2009, Multiscale finite element methods: Theory and applications: Springer, Surveys and tutorials in the applied mathematical sciences 4 .

Engquist, B., H. Holst, and O. Runborg, 2007, Multiscale methods for the wave equation: PAMM Proceedings in Applied Mathematics and Mechanics, 7, 1140903-1140904, doi: 10.1002/pamm.200700930.

Engquist, B., H. Holst, and O. Runborg, 2011, Multi-scale methods for wave propagation in heterogeneous media: Communications in Mathematical Sciences, 9, 33-56, doi: 10.4310/CMS.2011.v9.n1.a2

Fornberg, B., 1990, High-order finite differences and the pseudospectral method on staggered grids: SIAM Journal on Numerical Analysis, 27, 904-918, doi: 10.1137/0727052.

Fu, S., Y. Efendiev, K. Gao, and R. Gibson Jr., 2013, Multiscale modeling of acoustic wave propagation in $2 \mathrm{D}$ heterogeneous media using local spectral basis functions: 83rd Annual International meeting, SEG, Expanded Abstracts, 3553-3558

Gao, K., R. Gibson, Jr., E. Chung, Y. Efendiev, and S. Fu, 2013, A multiscale method for elastic wave equation modeling: 83rd Annual International meeting, SEG, Expanded Abstracts, 3444-3448.

Gibson, R., K. Gao, E. Chung, and Y. Efendiev, 2014, Multiscale modeling of acoustic wave propagation in 2D media: Geophysics, 79, no. 2, T61T75, doi: 10.1190/geo2012-0208.1

Goff, J. A., and T. H. Jordan, 1988, Stochastic modeling of seafloor morphology: Inversion of sea beam data for second-order statistics: Journal of Geophysical Research: Solid Earth, 93, 13589-13608, doi: 10.1029/ JB093iB11p13589.

Grechka, V., 2003, Effective media: A forward modeling view: Geophysics, 68, 2055-2062, doi: 10.1190/1.1635059.

Grechka, V., and M. Kachanov, 2006, Effective elasticity of rocks with closely spaced and intersecting cracks: Geophysics, 71, no. 3, D85D91, doi: 10.1190/1.2197489.

Helbig, K., and M. Schoenberg, 1987, Anomalous polarization of elastic waves in transversely isotropic media: Journal of the Acoustical Society of America, 81, 1235-1245, doi: 10.1121/1.394527.

Hobday, C., and M. Worthington, 2012, Field measurements of normal and shear fracture compliance: Geophysical Prospecting, 60, 488-499, doi: 10 $.1111 / j .1365-2478.2011 .01000 . x$.

Hudson, J. A., 1980, Overall properties of a cracked solid: Mathematical Proceedings of the Cambridge Philosophical Society, 88, 371-384, doi: $10.1017 /$ S0305004100057674.

Hughes, T. J., 1987, The finite element method: Linear static and dynamic finite element analysis: Dover Publications.

Kachanov, M., 1980, Continuum model of medium with cracks: Journal of the Engineering Mechanics Division, 106, 1039-1051.

Kachanov, M., 1992, Effective elastic properties of cracked solids: Critical review of some basic concepts: Applied Mechanics Reviews, 45, 304335, doi: $10.1115 / 1.3119761$.

Käser, M., and M. Dumbser, 2008, A highly accurate discontinuous Galerkin method for complex interfaces between solids and moving fluids: Geophysics, 73, no. 3, T23-T35, doi: 10.1190/1.2870081.

Klimeš, L., 2002, Correlation functions of random media: Pure and Applied Geophysics, 159, 1811-1831, doi: 10.1007/s00024-002-8710-2.

Komatitsch, D., J.-P. Vilotte, R. Vai, J. M. Castillo-Covarrubias, and F. J. Sánchez-Sesma, 1999, The spectral element method for elastic wave equations - Application to 2-D and 3-D seismic problems: International Journal for Numerical Methods in Engineering, 45, 1139-1164 doi: 10.1002/(SICI) 1097-0207(19990730)45:9<1139::AID-NME617>3 .0.CO;2-T.

Korostyshevskaya, O., and S. Minkoff, 2006, A matrix analysis of operatorbased upscaling for the wave equation: SIAM Journal on Numerical Analysis, 44, 586-612, doi: 10.1137/050625369.
Larson, M. G., and F. Bengzon, 2013, The finite element method: Theory, implementation, and applications: Springer, Texts in Computational Science and Engineering 10.

Marfurt, K., 1984, Accuracy of finite difference and finite element modeling of the scalar and elastic wave equations: Geophysics, 49, 533-549, doi: 10 $.1190 / 1.1441689$.

Owhadi, H., and L. Zhang, 2008, Numerical homogenization of the acoustic wave equations with a continuum of scales: Computer Methods in Applied Mechanics and Engineering, 198, 397-406, doi: 10.1016/j.cma 2008.08.012.

Rijpsma, G., and W. Zijl, 1998, Upscaling of Hooke's law for imperfectly layered rocks: Mathematical Geology, 30, 943-969, doi: 10.1023/A 1021729508736

Saenger, E., and T. Bohlen, 2004, Finite-difference modeling of viscoelastic and anisotropic wave propagation using the rotated staggered grid: Geophysics, 69, 583-591, doi: 10.1190/1.1707078.

Saenger, E. H., R. Ciz, O. S. Krüger, S. M. Schmalholz, B. Gurevich, and S. A. Shapiro, 2007, Finite-difference modeling of wave propagation on microscale: A snapshot of the work in progress: Geophysics, 72, no. 5 , SM293-SM300, doi: 10.1190/1.2753552.

Saenger, E. H., N. Gold, and S. A. Shapiro, 2000, Modeling the propagation of elastic waves using a modified finite-difference grid: Wave Motion, 31, 77-92, doi: 10.1016/S0165-2125(99)00023-2.

Sayers, C., and M. Kachanov, 1991, A simple technique for finding effective elastic constants of cracked solids for arbitrary crack orientation statistics: International Journal of Solids and Structures, 27, 671-680, doi: 10.1016/ 0020-7683(91)90027-D.

Sayers, C. M., 1995, Stress-dependent elastic wave velocities in shales: International Journal of Rock Mechanics and Mining Sciences and Geomechanics Abstracts, 32, 263-267, doi: 10.1016/0148-9062(94) 00049-9.

Sayers, C. M., 2002, Stress-dependent elastic anisotropy of sandstones: Geophysical Prospecting, 50, 85-95, doi: 10.1046/j.1365-2478.2002 $.00289 . \mathrm{x}$

Sayers, C. M., 2006, Sensitivity of time-lapse seismic to reservoir stress path: Geophysical Prospecting, 54, 369-380, doi: 10.1111/j.1365-2478 .2006.00539.x.

Sayers, C. M., and M. Kachanov, 1995, Microcrack-induced elastic wave anisotropy of brittle rocks: Journal of Geophysical Research, 100, 4149-4156, doi: 10.1029/94JB03134.

Schoenberg, M., and F. Muir, 1989, A calculus for finely layered anisotropic media: Geophysics, 54, 581-589, doi: 10.1190/1.1442685.

Schoenberg, M., and C. M. Sayers, 1995, Seismic anisotropy of fractured rock: Geophysics, 60, 204-211, doi: 10.1190/1.1443748.

Thomsen, L., 1986, Weak elastic anisotropy: Geophysics, 51, 1954-1966, doi: 10.1190/1.1442051.

Tsvankin, I., 2005, Seismic signatures and analysis of reflection data in anisotropic media 2nd ed.: Elsevier Science Publishing Co., Inc.

Tsvankin, I., J. Gaiser, V. Grechka, M. van der Baan, and L. Thomsen, 2010, Seismic anisotropy in exploration and reservoir characterization - An overview: Geophysics, 75, no. 5, 75A15-75A29, doi: 10.1190/1 .3481775 .

Tsvankin, I., and V. Grechka, 2011, Seismology of azimuthally anisotropic media and seismic fracture characterization: SEG.

Vdovina, T., and S. Minkoff, 2008, An a priori error analysis of operator upscaling for the acoustic wave equation: International Journal of Numerical Analysis and Modeling, 5, 543-569.

Vdovina, T., S. Minkoff, and O. Korostyshevskaya, 2005, Operator upscaling for the acoustic wave equation: SIAM Journal on Multiscale Modeling and Simulation, 4, 1305-1338.

Virieux, J., 1986, P-SV wave propagation in heterogeneous media: Velocitystress finite-difference method: Geophysics, 51, 889-901, doi: 10.1190/1 1442147.

Zijl, W., M. Hendriks, and C. 't Hart, 2002, Numerical homogenization of the rigidity tensor in Hooke's law using the node-based finite element method: Mathematical Geology, 34, 291-322, doi: 10.1023/A 1014894923280 . 